\title{
Optimal 3D Time-Energy Trajectory Planning for AUVs using Ocean General Circulation Models
}

\author{
Sultan Albarakati ${ }^{\mathrm{a}, *}$, Ricardo M. Lima ${ }^{\mathrm{a}}$, Thomas Theußl ${ }^{\mathrm{b}}$, Ibrahim Hoteit ${ }^{\mathrm{c}}$, Omar M. \\ Knio $^{\mathrm{a}}$ \\ ${ }^{a}$ Computer, Electrical, and Mathematical Sciences and Engineering Division, King Abdullah University \\ of Science and Technology, Thuwal, Saudi Arabia \\ ${ }^{b}$ King Abdullah University of Science and Technology (KAUST), Core Labs, Thuwal, 23955-6900, Saudi \\ Arabia \\ ${ }^{c}$ Physical Science and Engineering Division, King Abdullah University of Science and Technology, \\ Thuwal, Saudi Arabia
}

\begin{abstract}
This paper develops a new approach for solving optimal time and energy trajectory planning problems for Autonomous Underwater Vehicles (AUVs) in transient, 3D, ocean currents. Realistic forecasts using an Ocean General Circulation Model (OGCM) are used for this purpose. The approach is based on decomposing the problem into a minimal time problem, followed by minimal energy subproblems. In both cases, a non-linear programming (NLP) formulation is adopted. The methodology is first tested in idealized, steady, $2 \mathrm{D}$ settings, to verify the effectiveness of the method in addressing the multi-objective optimization problem. The scheme is then demonstrated for time-energy trajectory planning problems in the Gulf of Aden. In particular, the numerical experiments illustrate the capability of generating Pareto optimal solutions in a broad range of mission durations. In addition, the analysis also highlights how the methodology effectively exploits both the vertical structure of the current field, as well as its unsteadiness, namely to minimize travel time and energy consumption.
\end{abstract}

Keywords: Time-energy trajectory planning, Ocean General Circulation Model, Gulf of Aden, Pareto optimal solutions, unsteady ocean current.

\footnotetext{
${ }^{*}$ Email: sultan.albarakati@kaust.edu.sa

Email addresses: ricardo.lima@kaust.edu.sa (Ricardo M. Lima), thomas.theussl@kaust.edu.sa (Thomas Theußl), ibrahim.hoteit@kaust.edu.sa (Ibrahim Hoteit), omar.knio@kaust.edu.sa (Omar M. Knio)
} 


\section{Nomenclature}

\section{Sets, Indices}

$\mathcal{I} \quad$ indices for the time steps

$\mathcal{M}$ indices for the problems to be solved

$\mathcal{T}$ indices for time horizon

\section{Parameters}

$\chi \quad$ the spatial domain

$\Delta E \quad$ energy step (constant along energy axis)

$\Delta t \quad$ time step (constant)

$\mathbf{x}\left(t^{f}\right)$ final position vector

$\mathbf{x}\left(t_{0}\right)$ initial position vector

$\mathbf{x}^{f} \quad$ destination

$\mathbf{x}_{0} \quad$ starting point

$\bar{E}^{0} \quad$ energy value corresponding to the minimum travel time

$\bar{m}_{\max }$ number of problems to be solved in parallel

$\mathcal{M}^{j} \quad$ partition of the set $M$ into subsets, each with a number of elements $\bar{m}_{\max }$

$a_{\max z}$ upper bound on the acceleration of the vehicle relative to the current in the $z$ direction

$a_{\max }$ upper bound on the acceleration of the vehicle relative to the current in $x y$ plane

$E^{k, m}$ energy consumption of the AUV obtained from problem $m$ for the travel time $t^{f, k}$

$k_{\max }$ the number of additional travel times to build the Pareto-optimal solution curve

$N \quad$ number of grid points in the time discretization

$t_{0} \quad$ initial time grid point

$t_{i} \quad$ time grid point

$T_{\max }$ the maximum travel times to build the Pareto-optimal solution curve

$v_{\max _{z}}$ upper bound on the velocity of the vehicle relative to the current in the $z$ direction

$v_{\max }$ upper bound on the velocity of the vehicle relative to the current in $x y$ plane

$x_{\max }^{f} \operatorname{maximum} x$ coordinate of the destination target

$x_{\min }^{f}$ minimum $x$ coordinate of the destination target

$x_{\max }$ maximum $x$ coordinate of $\chi$

$x_{\min }$ minimum $x$ coordinate of $\chi$

$y_{\max }^{f}$ maximum $y$ coordinate of the destination target 
$y_{\min }^{f} \quad$ minimum $y$ coordinate of the destination target

$y_{\max }$ maximum $y$ coordinate of $\chi$

$y_{\text {min }}$ minimum $y$ coordinate of $\chi$

$z_{\max }^{f}$ maximum $z$ coordinate of the destination target

$z_{\min }^{f} \quad$ minimum $z$ coordinate of the destination target

$z_{\max } \operatorname{maximum} z$ coordinate of $\chi$

$z_{\min }$ minimum $z$ coordinate of $\chi$

\section{Variables}

$\mathbf{u}(\mathbf{x}(t), t))$ non-homogeneous varying ocean current field $\left(\left(u_{x}(\mathbf{x}(t), t), u_{y}(\mathbf{x}(t), t), u_{z}(\mathbf{x}(t)), t\right)\right)$

$\mathbf{a}(t)$ acceleration of the vehicle with respect to the current $\left(a_{x}(t), a_{y}(t), a_{z}(t)\right)$

$\mathbf{v}(t)$ instantaneous velocity of the AUV with respect to the current $\left(v_{x}(t), v_{y}(t), v_{z}(t)\right)$

$g(\mathbf{x})$ smoothed indicator function, which defines whether the vehicle is inside or outside the obstacle

$t^{f} \quad$ time to get to destination

\section{Introduction}

Autonomous Underwater Vehicles (AUVs) are used in a wide range of applications. In a civilian application context, these include coastal ecosystem monitoring, search and rescue tasks, scientific exploration of deep sea, oil and gas exploration, inspection of underwater installations, and management of shipping operations (Stommel, 1989; Blidberg et al., 1991; Aghababa, 2012). AUVs are employed in military applications, e.g. for surveillance of ocean vehicles and troops (Yuh, 2000; Soulignac et al., 2008; Lolla et al., 2012).

The successful operation of AUVs relies on the integration of capabilities arising from different areas, namely robotics, sensors and control, ocean science (modeling the environment where the AUVs act), networking and communications, statistics, and decisionmaking (off-line and on-line intelligence to adapt to unpredicted situations). All of these areas are relevant to accomplish missions in different applications. In this work, we focus specifically on trajectory planning of AUVs in complex environments.

Trajectory planning for AUVs is challenging due to the variability of ocean currents, and the complexity of the bathymetry in the ocean. A related constraint is the limitation of fuel or battery life when AUVs navigate in long missions. Therefore, there is a need 
to enhance the capability of vehicles to operate in the ocean, by either increasing the energy capacity (Crimmins et al., 2006; Hagen et al., 2007; Rynne and von Ellenrieder, 2010), and/or taking advantage of energy of the ocean current (Rao and Williams, 2009; Fernández-Perdomo et al., 2010; Smith et al., 2010; Subramani et al., 2016). Trajectory planning is thus essential to increase the energy autonomy of AUVs and to determine feasible trajectories from deployment locations to areas of operation (??).

There is significant amount of literature in 2D trajectory planning for AUVs (Wang et al., 2016; Pêtrès et al., 2007; Sun et al., 2010; Yilmaz et al., 2008; Wang et al., 2019; Warren, 1990; Soulignac, 2011; Garau et al., 2005; Lolla et al., 2012), however 3D trajectory planning is relatively less explored. 3D trajectory planning and the ocean state in which the AUV operates should be well characterized, so that safe and optimal decisions may be determined and communicated to the vehicle. One also needs to characterize the sub-sea terrain and other obstacles that might be encountered during the mission of the AUV.

There have been attempts to solve the 3D path planning problem for AUVs. Ataei and Yousefi-Koma (2015) investigated 3D optimal path planning for miniature AUVs, and determined a set of optimal paths that have to pass through several waypoints. The authors considered four criteria to evaluate the optimal path, namely total length of path, safety margin, smoothness of the planar motion, and gradient of diving. Liu et al. (2015) provided a 3D path planning method for AUVs using a modified firefly algorithm that incorporates an autonomous motion strategy to avoid cases of invalid regions. Aghababa (2012) estimated optimal paths by formulating a nonlinear optimal control problem, and applying evolutionary algorithms to compute its solution.

One of the most common objectives of trajectory planning for AUV is to minimize the total travel time of the journey. Fast marching method was applied by Pêtrès et al. $(2005,2007)$ to obtain the minimum time path in a given current field. Soulignac et al. (2009) used a novel approach, called symbolic wavefront expansion, to determine the path that minimizes the total travel time of the AUV. Level set methods (Lolla et al., 2012, 2014) were used to find the time-optimal path of the AUV by solving a particle tracking equation backward in time in the presence of the current. A mixed integer non-linear programming model and solution approach for 2D trajectory planning for problems with nonlinear flow fields to find the minimum time was proposed in (Wang et al., 2019). 
Another common objective of trajectory planning methods is minimize the energy consumption. A variety of approaches have been demonstrated to plan energy optimal paths for AUVs in the presence of a complex current field. In particular, the $\mathrm{A}^{*}$ method is widely used for AUVs path planning problems. Traditionally, the method deals with stationary fields (Carroll et al., 1992), and the algorithm is used for computing obstaclefree paths for AUVs by discretizing the space into cells. Lately, a modified version of $A^{*}$ was proposed to account for the effects of velocity fields around the vehicle and to minimize energy consumption (Garau et al., 2005, 2009; Koay and Chitre, 2013; Lee et al., 2015).

Recently, Niu et al. (2019) integrated several algorithms, namely Voronoi roadmap, Dijkstra's searching, coastline expanding, and genetic algorithm to tackle the challenges of determining energy-efficient paths in the presence of complex obstacles and spatially and temporally varying ocean currents.

Some studies considered a multi-objective approach to trajectory planning. Aghababa (2012) used the solution of optimal control problem with non-linear space equation to find time-energy optimal path in the presence of the static and energetic obstacles, in this work the current was not considered. Kularatne et al. (2016) presented an A* approach that generates 2D time-optimal and energy efficient trajectories in time-dependent flow fields. Subramani et al. (2015) and Subramani and Lermusiaux (2016) used stochastic optimization methodology in conjunction with a level-set approach to find energy-optimal paths of AUVs navigating in time-dependent fields.

In this paper, we develop a trajectory planning framework to simultaneously minimize travel time and energy consumption for AUVs operating in realistic, 3D, unsteady ocean current fields. To address the multi-objective optimization problem, a tailored $\epsilon$ constraint method is developed (Hwang and Masud, 1979) that for a given travel time determines the trajectory having minimal energy consumption. We start from the minimal travel time problem, ignoring the energy constraints, by adapting the methodology introduced in Albarakati et al. (2019) for this purpose. Specifically, the techniques used to provide smooth interpolants of static Ocean General Circulation Model (OGCM) velocity and obstacle data are extended to accommodate unsteady ocean currents. The extension involves the incorporation of suitable space-time interpolants into the optimization process. Starting from the energy unconstrained minimal time solution, our algorithm first 
attempts to locally minimize energy. Then a systematic exploration of a range of travel times, bounded from below by the unconstrained minimal travel time, is pursued. The exploration is based on discretizing the range of travel times using a fixed time step, and a parallel solution algorithm is implemented in order to find the minimum energy solution for each travel time and to explore a diversified set of initial conditions.

We first test the performance of the solution framework in idealized settings, and then assess its performance using realistic OGCM data. To simplify the analysis, we rely on an objective formulation in which the AUV energy expenditure consist of hydrodynamic losses only. Whereas the methodology can readily accommodate more elaborate, missionoriented, objective functions, the assumptions used enable us to perform straightforward comparison of different cases. Using this approach, numerical experiments are performed which illustrate the capability of generating Pareto optimal solutions. Analysis of these experiments highlights how the methodology effectively exploits both the vertical structure of the current field, as well as its unsteadiness, to minimize travel time and energy consumption.

This paper is organized as follows. The statement of optimal time-energy trajectory planning problem is discussed in Section 2. Section 3 introduces our decomposition approach to the solution of the multi-objective optimization problem, and outlines the NLP formulation adopted to determine Pareto-optimal solutions. In Section 4, we specify the setup of the case studies used to test the algorithm and demonstrate its performance. Results of the numerical implementations are discussed in Section 5. Section 6 summarizes the main conclusions.

\section{Problem Statement}

We consider the 3D time-energy trajectory planning problem for an AUV within realistic time varying current fields simulated by an OGCM. The objective of our formulation is to minimize travel time and the energy consumption of a single AUV from starting point to destination subject to obstacle avoidance as well as kinematic constraints. To account for the energy consumption, a simplified approach is adopted, based on the assumptions that (i) hydrodynamic energy losses correspond to a constant drag coefficient; and (ii) energy consumed by on-board instrumentation is ignored. Accordingly, we may rely on a reduced expression of the energy consumption obtained by dropping the drag 
coefficient, and express the problem as:

$$
\begin{array}{ll}
\min & \int_{t_{0}}^{T} 1 d t=T-t_{0}, \\
\min & \int_{t_{0}}^{T}\|\mathbf{v}(t)\|_{2}^{2} d t \\
\text { s.t. } & \frac{d \mathbf{x}(t)}{d t}=\mathbf{v}(t)+\mathbf{u}(\mathbf{x}(t), t), \\
& \frac{d^{2} \mathbf{x}(t)}{d t^{2}}=\mathbf{a}(t)+\nabla \mathbf{u}(\mathbf{x}(t), t) \cdot \frac{d \mathbf{x}(t)}{d t}+\partial_{t} \mathbf{u}(\mathbf{x}(t), t), \\
& \mathbf{x}\left(t_{0}\right)=\mathbf{x}_{0}, \\
& \mathbf{x}(T)=\mathbf{x}^{T}, \\
& \sqrt{v_{x}^{2}(t)+v_{y}^{2}(t)} \leq v_{\max }, \\
& \sqrt{a_{x}^{2}(t)+a_{y}^{2}(t)} \leq a_{\max }, \\
& \left\|v_{z}(t)\right\| \leq v_{\max _{z}} \\
& \left\|a_{z}(t)\right\| \leq a_{\max _{z}}, \\
& g(\mathbf{x}(t))>0, \\
& \mathbf{x}(t) \in \chi \subset \mathbb{R}^{3}, \\
& \mathbf{v}(t), \mathbf{a}(t) \in \mathbb{R}^{3}, \\
& T \in \mathbb{R},
\end{array}
$$

where $\mathbf{x}(t)=(x(t), y(t), z(t))$ denotes the instantaneous position of the AUV at time $t$, $\mathbf{u}(\mathbf{x}(t), t)=\left(u_{x}(\mathbf{x}(t), t), u_{y}(\mathbf{x}(t), t), u_{z}(\mathbf{x}(t)), t\right)$ is the time varying current field, $\mathbf{v}(t)=$ $\left(v_{x}(t), v_{y}(t), v_{z}(t)\right)$ is the instantaneous velocity of the AUV with respect to the current, and $\mathbf{a}(t)=\left(a_{x}(t), a_{y}(t), a_{z}(t)\right)$ is the acceleration of the vehicle with respect to the current. The total, ground-referenced, AUV velocity is $\mathbf{u}+\mathbf{v}$. The starting and ending time of the AUV trajectory are denoted by $t_{0}$ and $T$, respectively. The corresponding starting point and ending point are $\mathbf{x}\left(t_{0}\right)=\left(x_{0}, y_{0}, z_{0}\right), \mathbf{x}(T)=\left(x^{T}, y^{T}, z^{T}\right)$, respectively. The vehicle's velocity and acceleration relative to the current in the $x, y$ plane are bounded by $v_{\max }$ and $a_{\max }$; the maximum velocity and acceleration of the AUV relative to the current in the $z$ - direction are respectively bounded by $v_{\max _{z}}$ and $a_{\max _{z}}$.

To enforce obstacles avoidance, we introduce the smoothed indicator function $g$, which 
is defined on the problem domain $\chi$ according to:

$$
\begin{cases}g(\mathbf{x})>0, & \mathbf{x} \text { is in the fluid } \\ g(\mathbf{x}) \leq 0, & \text { otherwise }\end{cases}
$$

In order for the trajectory to avoid all obstacles, $g(\mathbf{x}(t))$ is required to be positive at all times, $t_{0} \leq t \leq T$. The constraint $\mathbf{x}(t) \in \chi$ forces the vehicle to navigate within the problem domain.

The formulation above, provides the continuous multi-objective function and constraints for solving the AUV trajectory planning problem, which is known as a complex NP-hard problem (Canny et al., 1991).

\section{Solution Approach}

We first present a time-discrete formulation of Problem (1) to obtain an amenable formulation for an optimization solver, and then describe the solution approach to obtain the Pareto-optimal solutions for the energy consumed vs travel time.

\subsection{Time-discretization for constraints}

Problem (1) describes the continuous objective functions and constraints for solving the AUV trajectory planning problem. Let $\mathcal{I}=\{0,1,2,3, \ldots, N\}$ be a set of indices, and $\mathcal{T}=\left\{t_{0}, t_{1}, t_{2}, \ldots, t_{N}\right\}$ be a collection of discrete times. We use $t_{0}=0$ as initial time, and assume that the total travel time $T \in \mathcal{T}$.

We use a time grid divided into steps of equal size, $\Delta t$. We thus have

$$
\Delta t=t_{1}-t_{0}=t_{2}-t_{1}=\ldots=t_{N}-t_{N-1} ; \quad t_{i}=i \cdot \Delta t, \quad i \in \mathcal{I} .
$$

The discrete version of the kinematic equations is derived based on a Taylor expansion at $t_{i}$. Specifically, we use:

$$
\begin{gathered}
\mathbf{x}\left(t_{i+1}\right) \approx \mathbf{x}\left(t_{i}\right)+\mathbf{v}\left(t_{i}\right)+\mathbf{u}\left(\mathbf{x}\left(t_{i}\right), t\right) \cdot \Delta t+\frac{1}{2} \mathbf{a}\left(t_{i}\right) \cdot \Delta t^{2}, \quad i \in \mathcal{I}, \\
\mathbf{v}\left(t_{i+1}\right) \approx \mathbf{v}\left(t_{i}\right)+\mathbf{a}\left(t_{i}\right) \cdot \Delta t, \quad i \in \mathcal{I},
\end{gathered}
$$


where the gradient of $\mathbf{u}$ with respect to space and time is ignored because the current is slowly changing at the resolution afforded by the OGCM simulation.

\subsection{Handling two objective functions}

After discretizing the constraints, one needs to address the multi-objective optimization problem, which admits a set of solutions (optimal energy consumption and travel time), named nondominated solutions or Pareto-optimal solutions (Hwang and Masud, 1979). Basically, for each travel time the corresponding minimum energy consumption is determined. These Pareto-optimal solutions are then represented in a chart that facilitates selection of a single solution (energy consumed and travel time).

Pareto-optimal curves are based on solutions of multiple optimization problems, and therefore, building a high resolution curve can be computationally demanding. Also, the decision-maker is faced with the selection of a solution from a curve, which may not be trivial to select or it may require other relevant criteria. To overcome these chcallenges, an alternative approach could be the determination of a single optimal solution, an ideal or utopian point (?), which then facilitates the solution selection, but may also involve computational challenges. This work investigates the trends of time-energy Pareto curves for real-world ocean currents in 2D and 3D. The objective is to study time-energy Pareto curves for problems involving currents that are transient and vary in the $x-y$ plane and with depth. Pareto-optimal solutions frequently exhibit monotonic curves, which in our case are represented by curves with the energy consumption decreasing with the travel time increasing. However, in time-energy trajectory problems, more elaborate Paretooptimal curves can be obtained. The analysis of the trends and trajectory solutions of these curves is conducted below. Note that in the present formulation the energy consumed by on-board sensors, actuators, and instrumentation is ignored. Thus, the Pareto curves presented neglect the contribution of this hardware, which varies with travel time.

The following sub-sections describe the problems solved and solution approach to build the Pareto-optimal curves. For that, we rely on some of the characteristics of the problem, namely that the set of travel times are the time grid points (greater than the minimum travel time). Therefore, we first calculate the minimum travel time from the starting point to the target neglecting the energy consumption objective function and constraints; and then for that travel time, the corresponding minimum energy is calculated. We repeat 
this calculation process for each (admissible) travel time.

\subsection{Minimum travel time and set of travel times}

To find the minimum travel time, the solution approach proposed in Albarakati et al. (2019) is used to solve a revised version of Problem (1) (including only the objective function for the travel time).

\subsection{Minimum energy for each travel time}

Based on the minimum travel time and the value of $\Delta t$, we construct a set of travel times defined by

$$
t^{f, k}=t^{f}+k \Delta t, \quad k=0,1, \ldots, k_{\max },
$$

where $t^{f}$ is the minimum travel time, $\Delta t$ is a time step as defined in Section 3.1, and $k_{\max }$ is the number of additional travel times to build the Pareto-optimal solution curve. We define the maximum time of interest for the trajectory planning as $T_{\max }=t^{f, k_{\max }}$, which sets the interval $\left[t^{f}, T_{\max }\right]$ of interest to calculate the Pareto-optimal solutions.

For each $t^{f, k}$, we are interested in finding the minimum energy consumption of an associated trajectory from the starting point to the target. To address this problem, an adapted version of the $\epsilon$-constraint method (Hwang and Masud, 1979) is used, whereby for each $t^{f, k}$, we solve a set of problems with $E \leq \bar{E}+m \Delta E, \forall m \in \mathcal{M}$, where $\bar{E}$ and $\Delta E$ are constants. $\bar{E}$ is described in detail later, and $\Delta E$ defines the resolution of the Pareto-optimal solutions. Therefore, to find the minimum energy for each fixed travel 
time $t^{f, k}, \forall k \in \mathcal{K}$, have one has to solve $|m|$ problems:

$$
\begin{aligned}
& \min _{\mathbf{x}, \mathbf{v}, \mathbf{a}, E} t^{f, k} \\
& \text { s.t. } \mathbf{x}\left(t_{i+1}\right)=\mathbf{x}\left(t_{i}\right)+\left(\mathbf{v}\left(t_{i}\right)+\mathbf{u}\left(\mathbf{x}\left(t_{i}\right)\right)\right) \Delta t+\frac{1}{2} \mathbf{a}\left(t_{i}\right) \Delta t^{2}, \quad \forall t_{i} \in \mathcal{T}, \\
& \mathbf{v}\left(t_{i+1}\right)=\mathbf{v}\left(t_{i}\right)+\mathbf{a}\left(t_{i}\right) \cdot \Delta t, \quad \forall t_{i} \in \mathcal{T}, \\
& \sqrt{v_{x}^{2}\left(t_{i}\right)+v_{y}^{2}\left(t_{i}\right)} \leq v_{\max }, \quad \forall t_{i} \in \mathcal{T} \\
& \sqrt{a_{x}^{2}\left(t_{i}\right)+a_{y}^{2}\left(t_{i}\right)} \leq a_{\max }, \quad \forall t_{i} \in \mathcal{T}, \\
& \left\|v_{z}\left(t_{i}\right)\right\| \leq v_{\max _{z}}, \quad \forall t_{i} \in \mathcal{T} \\
& \left\|a_{z}\left(t_{i}\right)\right\| \leq a_{\max _{z}}, \quad \forall t_{i} \in \mathcal{T}, \\
& g\left(\mathbf{x}\left(t_{i}\right)\right)>0, \quad \forall t_{i} \in \mathcal{T} \\
& E=\sum_{t_{i}=t_{0}}^{t^{f, k}}\left\|\mathbf{v}\left(t_{i}\right)\right\|_{2}^{2}, \\
& E \leq \bar{E}+m \Delta E \\
& \mathbf{x}\left(t_{0}\right)=\mathbf{x}_{0}, \\
& \mathbf{x}\left(t^{f, k}\right)=\left(x^{t^{f, k}}, y^{t^{f, k}}, z^{t^{f, k}}\right), \\
& \mathbf{x}\left(t_{i}\right) \in \chi \subset \mathbb{R}^{3}, \quad \forall t_{i} \in \mathcal{T} \\
& \mathbf{v}\left(t_{i}\right), \mathbf{a}(t) \in \mathbb{R}^{3}, \quad \forall t_{i} \in \mathcal{T} \\
& E \in \mathbb{R}_{+} .
\end{aligned}
$$

Problem (7) is a constrained nonlinear system with a fixed objective function $t^{f, k}$ and a set of trajectory constraints derived according to the discretization of Section 3.1, and an upper bound on the energy consumption of the AUV. Let $E^{k, m}$ be the energy consumption of the AUV resulting from problem $m$ for the travel time $t^{f, k}$, then the minimum energy consumption of the AUV for $t^{f, k}$ is $E^{k}=\min _{m}\left\{E^{k, m}\right\}$.

\subsection{Solution approach to solve (7)}

In the present setup, the Pareto-optimal trajectories are built for a nonlinear flow that is time and space dependent. This may lead to a complex, non-monotonic Pareto curve. The Pareto-optimal solutions may exhibit alternative trends depending on whether the vehicle is traveling along the current or against it and the relative difference between the maximum relative velocity of the AUV and the velocity of the current, which is not 
straightforward to assess, given the 3D transient ocean environment. Also, as the $t^{f, k}$ increases, the size of the problems in (7) also increases in terms of number of variables and constraints.

To overcome such complexities, a solution approach is proposed that accommodates nonmonotonic Pareto curves. Two procedures are considered, depending on the value of $k$ : one for $k=0$ and another for $k>0$.

For $k=0$, which corresponds to the minimum travel time found, the minimum value of energy is always less or equal than the energy value corresponding to the minimum travel time, denoted as $\bar{E}^{0}$. Therefore, we have $\bar{E}=\bar{E}^{0}$ and the set $\mathcal{M}$ is defined as

$$
m \in\left\{-m_{\max } ;-m_{\max }+1 ; \ldots ;-1\right\}
$$

which means that for $t^{f, 0}$, Problem (7) is solved for a set of decreasing upper bounds on the energy. If the problems were solved sequentially, this process would terminate when for a given $m$ the problem is infeasible, and therefore, the minimum energy would be $E^{k}=E^{k, m-1}$.

For $k>0, \mathcal{M}$ is defined to account for the case where the minimum energy for $t^{f, k}$ can be greater or less than the energy $E^{k-1}$ from $t^{f, k-1}$ (this is illustrated in the results). Thus, $\mathcal{M}$ is defined as

$$
m \in\left\{-\frac{m_{\max }-1}{2}, \ldots,-1,0,1, \ldots, \frac{m_{\max }-1}{2}\right\}
$$

where $m_{\max }$ is assumed to be an odd number. At the end of the solution of all problems in (7), we get the Pareto-optimal solutions, namely the pairs $\left(t^{f, k}, E^{k}\right)$.

In the above general definitions of $\mathcal{M}$, this set can be large and a sequential solution of the problems would be computationally demanding. Therefore, we propose a parallel implementation, whereby, for each $t^{f, k}$, we partition the set $\mathcal{M}$ into subsets $\mathcal{M}^{j}$, each with a number of elements $\bar{m}_{\max }$, where $\bar{m}_{\max }$ corresponds to the number of problems to be solved in parallel. This implementation is presented in Algorithm 1 and Algorithm 2, and illustrated in Figure 1 with an example of a set of problems solved for $t^{f, k}, k=$ $0,1,2$, and 3 . 


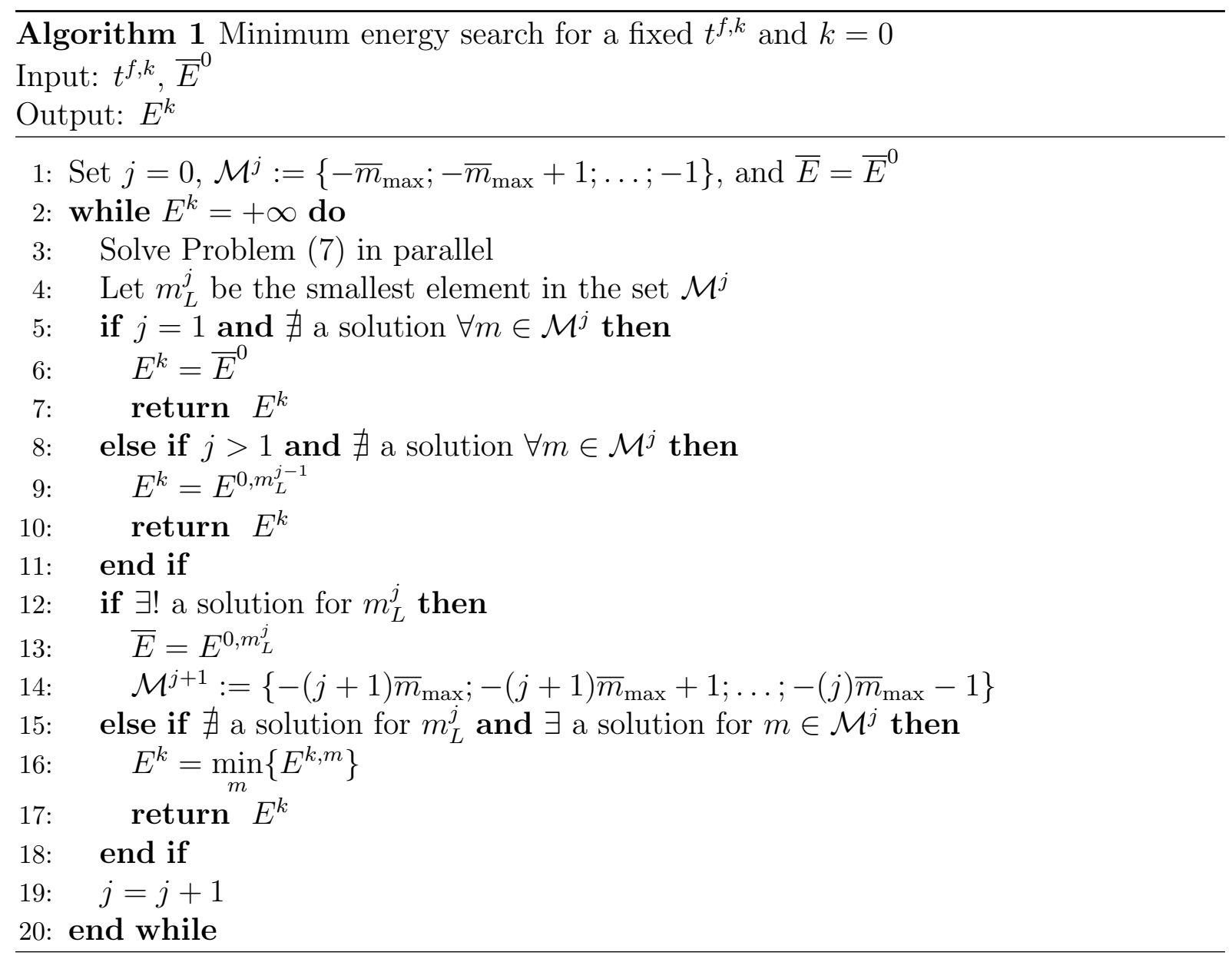

\subsection{Remarks}

- Previously computed solutions are utilized to initialize the solver and reduce the computational time required to solve each problem. For example, the solution from the minimum travel time problem is used to initialize the problems solved in parallel for $k=0$, for the first $\mathcal{M}^{j}$. In the same fashion, the solution from the Problem (7) corresponding to the minimum energy for $t^{f, k-1}$ is used to initialize all problems for $t^{f, k}$.

- We assume that if the solver does not return a solution for a given problem $(m, k)$, then the problem is infeasible.

\subsection{Diversification strategy to find additional Pareto-optimal solutions}

The problems defined in (7) are constrained nonlinear problems that are non-convex due to the nature of the flow field obtained from the OGCM data. Therefore, to make an effort to find additional solutions for the problems that return infeasible solutions, a 


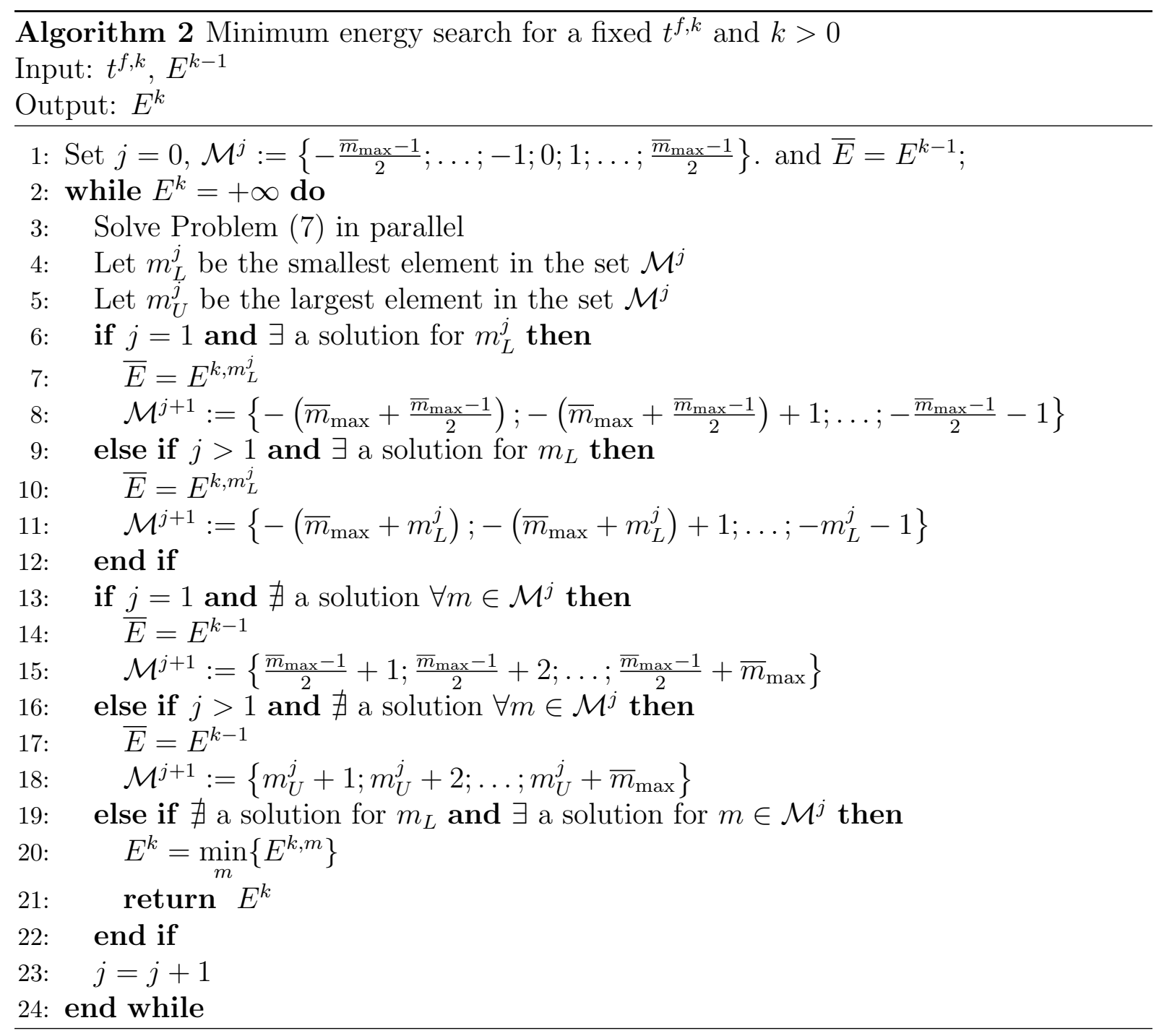

diversification strategy is implemented that provides different initialization solutions. The goal is to robustify the overall solution approach.

The diversification strategy randomly samples a specified number of waypoints, $n_{w}$, in the space. Each waypoint corresponds to one initialization and only one waypoint per trajectory is fixed. As a result, we generate $n_{w}$ initial paths to the target that are used to initialize the problems in (7). Therefore, we run the solution approach described before for each initialization path with a specified waypoint and one run without waypoint. We build the Pareto-optimal solution curves over all waypoints solutions. This diversification approach is illustrated in the flowchart in Figure 2.

\subsection{Implementation Details}

We implemented Problem (7) and the solution approach in the modeling system GAMS (GAMS, 2018). Problem (7) was solved with IPOPT 3.12 (Wächter and Biegler, 2006) 
and the optimization runs were performed on a workstation with 24 logical CPUs, each with $2.5 \mathrm{GHz}$ and $128 \mathrm{~GB}$ the total RAM. The stopping criteria for the solution of each set of problems solved in parallel defined by $\mathcal{M}^{j}$ is 100 seconds for Case 1 and Case 2, and 300 seconds for Case 3 and Case 4.

\section{Numerical Experiments}

To demonstrate the proposed algorithm, and to study the behavior of Pareto curves in controlled settings, we first consider two simplified cases involving idealized, steady, current fields. The first case involves a steady uniform current, which we refer to as Case 1. The second case, Case 2, involves a current exhibiting a vertical shear. Next, we consider two cases on a realistic setting based on an unsteady OGCM flow. In the first, Case 3, the AUV is restricted to move along the surface; in the second, Case 4, the restriction is relaxed so that the optimization considers the full 3D transient OGCM field. This section, provides a brief description of the current field in each of these four cases. The results of these experiments are presented in the following section.

\subsection{Case 1}

As summarized in Table 1, the first example has uniform favorable, steady current in the problem domain without any obstacles. The (normalized) magnitude of the current velocity is 1 , and the maximum relative velocity of the vehicle is 0.5 .

\subsection{Case 2}

In this case, also summarized in Table 1, a time-independent current is considered which varies along the $z$ direction only, and is uniform in $x$. The vertical profile is assumed to be given by:

$$
u_{x}(z, t)=\exp \left(-\alpha\left(z-z_{0}\right)^{2}\right)
$$

As specified below, two separate instances are considered in this case, corresponding to favorable and adverse currents.

\subsubsection{Instance $I$}

In this Instance, exponentially decaying favorable current (8) is considered, with $\alpha=$ $1 / 100$ and $z_{0}=50$, from the straight line joining the starting point to the end point. There are no obstacles in the domain of the problem. The magnitude of the current is 
defined in the interval $[0,1]$, see Figure 5 , and the maximum magnitude of the relative velocity of the vehicle is 0.5 .

\subsubsection{Instance II}

In this Instance, exponentially decaying unfavorable current (8) is considered, with $\alpha=1 / 100$ and $z_{0}=50$, from the straight line joining the starting point to the end point. There are no obstacles in the problem domain. The magnitude of the current is defined in the interval $[0,1]$; and the maximum magnitude of the relative velocity of the vehicle is 1 .

\subsection{Time-dependent $O G C M$ field}

The realistic time-dependent ocean fields were simulated using an assimilative OGCM of the Red Sea (Toye et al., 2017, 2018). The underlying assimilation system operates sequentially as cycles of forecast-analysis steps, using the MIT ocean general circulation model (MITgcm) for forecasting the Red Sea circulation, and an ensemble Kalman filter (EnKF) for updating the model forecasts with satellite sea surface height and temperature observations every time they become available (Hoteit et al., 2013). The system domain extends from $30^{\circ} \mathrm{E}$ to $50^{\circ} \mathrm{E}$ and from $10^{\circ} \mathrm{N}$ to $30^{\circ} \mathrm{N}$, covering the whole Red Sea, the Gulf of Suez, the Gulf of Aqaba, and the Gulf of Aden. To measure Euclidean distance in the problem domain, we consider $30^{\circ}$ longitude, $10^{\circ}$ latitude, and the sea level as the origin, and measure all the distances from this point. The system was configured on a spherical grid with a $0.04^{\circ} \times 0.04^{\circ}$ horizontal resolution, resulting in $500 \times 500$ grid points, and 50 vertical layers ranging from $4 \mathrm{~m}$ at the surface to $300 \mathrm{~m}$ near the bottom. The model bathymetry was extracted from the gridded General Bathymetric Chart of the Ocean (GEBCO), and the model was forced with 6-hourly atmospheric fields from the European Centre for Medium-Range Weather Forecasts (ECMWF) reanalysis. The eastern open boundary conditions in the Gulf of Aden include velocities, temperature and velocity fields and were obtained from the German contribution of the Estimating the Circulation and Climate of the Ocean project (GECCO). After validating the model outputs with available in situ and satellite remote sensing observations (Yao et al., 2014b,a), this ensemble assimilation system was integrated to generate flow-fields conditioned for the month of January 2006. From this data set, we selected the snapshots from January 19, January 22, and January 25, corresponding to the assimilated solution (ensemble mean over three 
days each).

Bathymetry and current functions were extracted from this data spatially with the tri-cubic method also used in Albarakati et al. (2019). The current functions were also interpolated linearly in time.

\subsection{Case 3}

In Case 3, we shift our focus to use time dependent current and a realistic OGCM simulation field. First, we study a 2D case in the Gulf of Aden. This corresponds to an AUV moving on the surface of the ocean. The selected region for the problem is illustrated in Figure 7. The figure also shows the waypoints used for initialization and the initialization paths.

\subsubsection{Instance $I$}

In this instance, the start and end point of the AUV are chosen so that the current is mostly favorable.

\subsubsection{Instance $I I$}

In this instance, the start and end point of the AUV are flipped so that the current is mostly unfavorable.

\subsection{Case 4}

In Case 4, we study the same problem as in Case 3 but relax the sea surface constraint, so the vehicle can also take advantage of diving.

\subsubsection{Instance $I$}

In this instance the start and end point of the AUV are again chosen so that the current is mostly favorable.

\subsubsection{Instance $I I$}

In this instance the start and end point of the AUV are again chosen so that the current is mostly unfavorable.

Table 2 lists the parameters of Cases 3 and 4, including the values of the start and end points of the AUV journey, details of the spatial domain, time horizon, and distance between start and end point. 
Note that for Cases 3 and 4, the maximum mission duration specified in Table 2 is approximately 6 days, and one would generally require that this falls with the time horizon over which the operational ocean forecast can be considered reliable. Though this time horizon may depend on a variety of factors, including resolution, quality of observations used in assimilation, frequency of observations or measurements, as well as prevailing dynamical features of the local circulation, with state-of-the-art models (Chassignet et al., 2018) and suitable resolution, OGCMs may be generally anticipated to provide reliable forecasts for horizons of 1-3 weeks, i.e. over time periods that are larger than $T_{\max }$.

\section{Results}

This section presents the results of the optimization experiments for the case studies described above. The computational cost of the algorithms and the size of the models are summarized in Table 3. Advanced visualization tools are used to analyze and amplify optimization results. The discussion follows each case individually.

\subsection{Case 1}

The Pareto curve of the numerical solution of Case 1 is shown in Figure 3, which also shows the Pareto curve of the analytical solution of this problem. To estimate the latter, we first note that because we have a uniform favorable current along the straight line from the starting point to destination, and because we are minimizing energy and time, all optimal paths are straight lines. Intuitively, the only reason the AUV would veer off from a straight path will be to reach the target with a time delay, but the same can be achieved by decreasing the velocity while simultaneously consuming less energy. Hence straight paths are always optimum. In addition, because the energy is a quadratic functional of the relative velocity, $v_{x}$, we assume that $v_{x}$ is steady ${ }^{1}$, and thus estimate the energy, $E(T)$, corresponding to travel time, $T$, simply according to:

$$
E(T)=v_{x}^{2} T
$$

The travel time $T$ may be expressed according to:

$$
T=\frac{D_{\mathbf{x}_{0} \rightarrow \mathbf{x}^{f}}}{v_{x}+u_{c}}
$$

\footnotetext{
${ }^{1} v_{x}$ is assumed constant only in this analysis, in the remaining problems it is a variable.
} 
where $u_{c}=1$ is the current velocity, and $D_{\mathbf{x}_{0} \rightarrow \mathbf{x}^{f}}$ is the distance between the starting point and the target. Consequently, for the present 1D example, we have:

$$
E(T)=\left(\frac{D_{\mathbf{x}_{0} \rightarrow \mathbf{x}^{f}}}{T}-u_{c}\right)^{2} T,
$$

with $T$ in the range

$$
\frac{D_{\mathbf{x}_{0} \rightarrow \mathbf{x}^{f}}}{u_{c}+v_{\max }} \leq T \leq \frac{D_{\mathbf{x}_{0} \rightarrow \mathbf{x}^{f}}}{u_{c}-v_{\max }}, \quad \text { with } u_{c}-v_{\max }>0
$$

where $v_{\max }$ is the maximum AUV velocity. For the parameters specified in Section 4.1 and Table 1, the estimates above indicate that there is a zero energy solution around time 80, which is the time it takes the current to transport the vehicle from its starting point to the target. For shorter or longer travel times, the vehicle has to spend energy to either speed up or slow down. This also means, that before time 54 and after time 160 there are no feasible solutions.

Figure 3 shows that the numerical results are in close agreement with the analytical solution, in the entire range of feasible travel times. This demonstrates that, in the present case involving a convex problem, the algorithm yields the global optima.

\subsection{Case 2}

In this case we consider two instances.

\subsubsection{Instance $I$}

In Instance I, a favorable current field is considered which has vertical shear as given by Equation 8. The Pareto curve of the numerical solution is shown in Figure 4a, and the paths for a few sampled points on the Pareto curve in Figure 5. Note that as the travel time increases from its minimum value, the energy spend monotonically decreases, and vanishes at $t^{f}=87$. For this travel time, the AUV does not consume energy, as the current transports to its destination. Also note that for travel times ranging from the minimum to $t^{f}=87$, the trajectories correspond to a straight line path, for which energy consumption increases as travel time decreases.

For travel times $t^{f}>87$, the AUV may oppose the favorable current and follow a straight line to the target, which requires more energy. Alternatively, the AUV can dive towards regions of weaker current, which will increase the travel time without requiring 
consuming energy to fight the favorable current. Hence, as we increase time past the value $t^{f}=87$, the vehicle diverts from the straight line path and the deviation increases as the travel time increases.

\subsubsection{Instance $I I$}

Instance II is designed by simply switching the starting point and the target of the AUV journey. Because of this, the current is now adverse, with the strongest adverse effect occurring along the straight path joining the starting point and the target. The Pareto curve for the numerical solution of this problem is given in Figure 4b. Figure 6 presents the paths for a few sampled points on the Pareto curve. Notice that since we are minimizing both time and energy, the optimum path for the vehicle would always avoid regions of strong adverse current. In this instance, this corresponds to diving lower depth. As can be seen in Figure 6, as the travel time increases, the AUV dives to greater depths to reach a region of weak adverse currents. In fact, almost all the initial energy consumed by the AUV is used to move away from the region of strong adverse current, even if it means moving away from the target. Notice from Figure $4 \mathrm{~b}$ that as the travel time increases from its minimum value, the energy first drops rapidly, but then exhibits milder decay as the travel time becomes larger. This is consistent with the fact that in this case adverse current intensity drops exponentially with depth.

\subsection{Case 3}

In Cases 3 and 4, the domain in which the AUV is allowed to navigate is a region located in the Gulf of Aden; see Figure 7. In both cases, the starting point and target are at the ocean surface. In Case 3, the motion of the AUV is restricted to the ocean surface; this restriction is relaxed in Case 4.

An OGCM simulation for a two-week period starting 19 January, 2006 is used to describe the unsteady ocean current. As illustrated in Figure 8, the flow is characterized by strong eddy variability (Yao and Hoteit, 2016). This flow enables us to test the performance of the solution algorithm in conditions involving significant spatial and temporal ocean current variability.

\subsubsection{Instance $I$}

In this first instance, for most of its journey the AUV navigates in a favorable current, except for a strong eddy close to the target region (see Figure 9). The strength of this 
eddy decreases with time, and the current magnitude drops from around $1.56 \mathrm{~m} / \mathrm{s}$ to around $0.7 \mathrm{~m} / \mathrm{s}$, over the period 19 January 2006 to 25 January, 2006 (Figures 9a-9d)

An interesting point to notice is the slight dip in the paths right before the AUV reaches the vortex region (marked with $\mathrm{A}$ in Figure 9b). This dip is explained by several factors, namely a) the opposite directions of the current on the left and right sides of the region marked with $\mathrm{A}$; b) the low relative velocities in the trajectories with lower energy consumption; but also c) as a response of the trajectory planning to the transient strong current of the vortex region. Note that along the straight line path from the starting point to the destination, there are strong currents at earlier times, see Figure 9a, and at later times, see Figure 9d, with a time window with weaker currents between the 42 hours and 98 hours, approximately. The trajectory obtained for the minimum travel time reaches the vortex after strong currents have passed, see Figure 9b. Whereas, the trajectories obtained with higher travel times and lower energy consumption arrive later to the vortex region, taking advantage of the time window with weaker currents, see Figure 9c. However, because of the lower relative velocity of the AUV, the vortex current still induces the steer of the AUV resulting in the dip of the path.

Figure $9 \mathrm{e}$ shows that in the trajectory obtained with the minimum travel time, the magnitude of the relative velocity of the AUV is at the maximum value during all the travel time. Towards the end of the journey (around $42 \mathrm{hrs}$ ), when the AUV arrives at the vortex, the magnitude of the current velocity becomes higher, but it does not add much to the magnitude of the total velocity. These values of the magnitude of the total velocity mean that the AUV goes through the strong vortex almost perpendicularly, see Figures $9 \mathrm{~b}$ and $9 \mathrm{e}$.

The trajectory obtained with the minimum energy consumption has a low magnitude of the relative velocity and a lower magnitude of the total velocity, see Figures 9f, compared with the minimum travel time solution. However, when the AUV reaches the vortex, it almost glides to the destination taking advantage of the vortex current. This gliding effect is observed through the analysis of the magnitude of the total velocity, which increases substantially when the vehicle reaches the currents of the vortex, see Figures 9c, 9d and 9f.

A composite animation of Pareto-optimal trajectories, including the minimum time and minimum energy solutions, and surface current is made available in the supplementary 
material.

\subsubsection{Instance $I I$}

Case 3 Instance II is defined by interchanging starting point and destination Case 3 Instance I. This interchange makes the current along the straight line between these two points mostly unfavorable, which is translated into a higher minimum travel time in this case, $84 \mathrm{~h}$, compared with $58 \mathrm{~h}$ in Instance I. In addition, the paths obtained for the different travel times and energy consumption are highly influenced by the strong currents in the vortex, see Figure 10. The paths shown in Figure 10, start by the AUV moving to the left and down and then right taking advantage of the central current, see Figure 10a, after exiting the vortex, the AUVs adapt their path to take advantage of non-adverse currents to approach and arrive to the destination from the bottom, see Figure 10b.

Figure 10c, shows that in the trajectory obtained with the minimum travel time, the magnitude of the relative velocity of the AUV is mostly at the maximum value, but the magnitude of the total velocity drops below the relative velocity due to adverse currents. The trajectory corresponding to the minimum energy consumption exhibits higher and more volatile magnitudes of the relative velocity of the AUV than observed in Instance I. For both instances, the energy consumptions for the travel times equal to $120 \mathrm{~h}$ and $145 \mathrm{~h}$ show a considerable difference, 12 and 5 in Instance I versus 49 and 38 in instance II. These energy consumption differences result also from the adverse currents in Instance II. A composite animation of Pareto optimal trajectories, including the minimum time and minimum energy solutions, and of the surface current is also given in the supplementary material.

The Pareto curves for Case 3, Instances I and II, are presented in Figure 11. For Instance I the Pareto curves for all waypoints are similar and are almost monotonic, and for Instance II, there are minor differences between Pareto curves for different waypoints. As mentioned before, Instance I exhibits a smaller minimum travel time and for the same travel times lower levels of energy consumed.

\subsection{Case 4}

Case 4 is a 3D case in the Gulf of Aden region, where the AUV can travel at any depth between the sea surface and the sea floor. We have tested two instances for this case, both corresponding directly to the instances of Case 3 (Section 4.5), where the AUV 
was constrained to the sea surface.

\subsubsection{Instance $I$}

Modeling the ability of the AUV to exploit the depth dimension has an impact on the size and complexity of the optimization problems, as well as on the results. Although it is difficult to make definitive claims about the global optimality of a trajectory planning solution in the presence of nonlinear time varying current fields, considering the depth dimension allows the exploration of regions of more favorable currents.

The trajectories for the minimum time and minimum energy consumption are shown in Figures 12 and 13, respectively. The trajectory obtained for the minimum travel time shows a top view path similar to the one in Case 3, Instance I, but in this case the trajectory exhibits substantial depth variation; see Figure 14a for a comparison. However, the travel time in Case 4 is slightly greater than in Case 3, 60 hours versus 58 hours.

The minimum energy consumption solution also leads to a trajectory with depth variations, but the results show similar trends as in Case 3, namely in terms of the top view path, travel time, and energy consumption. See Figure 14b for a comparison.

Animations of the AUV trajectory and the ocean current for the minimum time and minimum energy solutions for Case 4 Instance I are provided in the supplementary material.

\subsubsection{Instance $I I$}

This instance is the mirror version of Case 4, Instance I, where we have swapped the starting point and destination of the AUV journey. The trajectories for the minimum time and minimum energy consumption are shown in Figures 15 and 16, respectively.

In this instance, we see a clear impact of the depth dimension in the trajectory results and Pareto-optimal solutions. Given the locations of the starting point and destination, and the adverse, time and depth varying, currents at three locations: a) the surface; b) along the straight line between starting point and destination; and c) along the path from Case 3, Instance II; the trajectories are completely distinct from Case 3 and Case 4, Instance I. The trajectories are different in terms of path, travel time, energy consumption, and depth. In this instance, the results show that the proposed optimization framework is able to find solutions that take advantage of the degrees of freedom available.

In the minimum time solution, we observe a different top view path, lower travel times, 
and lower energy consumption when comparing with the solution from Case 3, Instance II. Specifically, the top view path is distinct from the top view path from Case 3, Instance II; see Figure 14c for a comparison. The travel time is 62 hours in Case 4 versus 84 hours in Case 3, and the energy consumption in Case 4 is 60 versus 82 in Case 3. The depth of the trajectory is below $500 \mathrm{~m}$ most of the travel time, which is lower than in Case 4, Instance I, to avoid adverse currents.

In the minimum energy consumption solution, we observe a different top view path, and a lower energy consumption comparing to the same travel time of Case 3, Instance II. The top view path reported in Case 4 is distinct from that from Case 3; see Figure 14d for a comparison. The energy consumption in Case 4 is 23 versus 38 in Case 3.

Animations of the AUV trajectory and the ocean current for the minimum time and minimum energy solutions for Case 4 Instance II are provided in the supplementary material.

Figures 17a and 17b, show the Pareto curves for Case 4 Instances I and II, respectively. We Observe that whereas the Pareto curves from different waypoints of Case 3 (Figure 11) are all similar, adding waypoints has a significant effect on the solutions in Case 4. This is because with the addition of the depth axis, the size of the domain increases and many local minima appear. Also notice that the solution of the case of no waypoint is the worst amongst all solutions. We conclude that adding waypoints is essential in 3D scenarios to obtain reasonable solutions.

Figure 18a compares the best solutions (minimum energy across all waypoints for each time) for the $2 \mathrm{D}$ and $3 \mathrm{D}$ cases. Theoretically, the $3 \mathrm{D}$ solutions are equal or less than the $2 \mathrm{D}$ solutions for all values of time, since the $2 \mathrm{D}$ domain is a subset of the $3 \mathrm{D}$ domain. However, the $3 \mathrm{D}$ solutions are slightly worse than the 2D solutions. We ascribe this to the fact that with favorable currents the 2D solutions at the surface are good solutions, and thus, the third dimension does not improve the solution; and to the complexity of the problem and the existence of local minima in the 3D case.

In contrast, comparing Instance II of Case 3 and Case 4, see Figure 18b, the 3D solutions are much better than the $2 \mathrm{D}$ solutions, as discussed above. This is because the current is mostly adverse, so the optimization framework can find better solutions taking advantage of the depth axis to move the trajectory to regions of low adverse currents in the $3 \mathrm{D}$ case. 


\section{Conclusions}

We developed a new approach to determine time-energy optimal trajectories using realistic, 3D, time-dependent, OGCM data. In the proposed NLP approach, we first solve the original problem for the minimum travel time without the energy objective function and constraints; and then for each travel time we minimize the energy consumption. Parallelization and diversified initialization strategies are incorporated in order to reach solutions efficiently and to avoid local minima. The overall approach enables to determine the time-energy Pareto-optimal curve for complex problems.

The proposed framework was initially tested using idealized scenarios involving steady currents. This includes a 1D example admitting a closed form solution, as well as a 2D example involving a current field with a vertical shear. The results show that even for small cases, non-monotonic Pareto-optimal curves can be obtained depending on the maximum magnitude of the velocity of the AUV and the current, and relative position of the starting point and destination and direction of the flow.

The method was then applied to realistic 2D and 3D scenarios using OGCM simulations of the oceanic circulation in the Gulf of Aden. Results of the numerical experiments show the capability of the method to generate Pareto-optimal solutions in a broad range of travel times. Detailed visualization experiments are used to examine the predicted Pareto-optimal solutions. These visualizations illustrate how the AUV trajectory (path, velocity, and depth) adapts to the 3D structure of the current field, as well as its unsteadiness, to minimize travel time and energy consumption. The specific case of navigating against surface currents with starting point and destination at the surface highlights the capability of the optimization approach to find better solutions in a 3D structure than in a 2D. In this case, the trajectories take advantage of the third dimension to find more favorable currents and minimize travel time and energy consumption. The Pareto-optimal curves for the realistic scenarios are approximately monotonic due to the maximum time of interest considered, but also due to the characteristics of the current and velocity of the AUV considered.

Future work will focus on extending the present framework along different directions. This includes generalizing the formulation to account for forecast uncertainty, and accordingly implement risk-aware planning. A particularly attractive approach consists in exploiting ensemble-based methods (Wang et al., 2016; ?; ?; ?), which provide a nature 
means to capitalize information afforded by ensemble OGCM forecasts. Other avenues that are worthwhile exploring include fast online replanning following data assimilation and forecast updates, as well as incorporation of more elaborate objective functions particularly to include specific mission goals.

\section{Acknowledgment}

Research reported in this publication was supported by research funding from King Abdullah University of Science and Technology (KAUST), and used resources of the KAUST Core Labs.

Aghababa, M. P., 2012. 3D path planning for underwater vehicles using five evolutionary optimization algorithms avoiding static and energetic obstacles. Applied Ocean Research 38, $48-62$.

Albarakati, S., Lima, R. M., Giraldi, L., Hoteit, I., Knio, O., 2019. Optimal 3D trajectory planning for AUVs using ocean general circulation models. Ocean Engineering 188.

Ataei, M., Yousefi-Koma, A., 2015. Three-dimensional optimal path planning for waypoint guidance of an autonomous underwater vehicle. Robotics and Autonomous Systems 67, $23-32$.

Blidberg, D. R., Turner, R. M., Chappell, S. G., 1991. Autonomous underwater vehicles: Current activities and research opportunities. Robotics and Autonomous Systems 7 (2), $139-150$.

Canny, J., Rege, A., Reif, J., 1991. An exact algorithm for kinodynamic planning in the plane. Discrete \& Computational Geometry 6 (5), 461-484.

Carroll, K. P., McClaran, S. R., Nelson, E. L., Barnett, D. M., Friesen, D. K., William, G. N., 1992. AUV path planning: an A* approach to path planning with consideration of variable vehicle speeds and multiple, overlapping, time-dependent exclusion zones. In: Autonomous Underwater Vehicle Technology, 1992. AUV '92., Proceedings of the 1992 Symposium on. pp. 79-84.

Chassignet, E. P., Pascual, A., Tintoré, J., Verron, J. (Eds.), aug 2018. New Frontiers in Operational Oceanography. GODAE OceanView. 
Crimmins, D. M., Patty, C. T., Beliard, M. A., Baker, J., Jalbert, J. C., Komerska, R. J., Chappell, S. G., Blidberg, D. R., 2006. Long-endurance test results of the solar-powered AUV system. In: OCEANS 2006. pp. 1-5.

Fernández-Perdomo, E., Cabrera-Gámez, J., Hernández-Sosa, D., Isern-González, J., Domnguez-Brito, A. C., Redondo, A., Coca, J., Ramos, A. G., Fanjul, E. A., Garca, M., 2010. Path planning for gliders using regional ocean models: Application of Pinzó path planner with the ESEOAT model and the RU27 trans-atlantic flight data. In: OCEANS'10 IEEE SYDNEY. pp. 1-10.

GAMS, 2018. General Algebraic Modeling System (GAMS) Release 25.1.2. Washington, DC, USA.

URL http: //www.gams.com

Garau, B., Alvarez, A., Oliver, G., 2005. Path Planning of Autonomous Underwater Vehicles in Current Fields with Complex Spatial Variability: an A* Approach. In: Proceedings of the 2005 IEEE International Conference on Robotics and Automation. pp. 194-198.

Garau, B., Bonet, M., Alvarez, A., Ruiz, S., Pascual, A., 2009. Path planning for autonomous underwater vehicles in realistic oceanic current fields: Application to gliders in the Western Mediterranean Sea. Journal of Maritime Research 6 (2), 5-22.

Hagen, P. E., Midtgaard, O., Hasvold, O., 2007. Making AUVs truly autonomous. In: OCEANS 2007. pp. 1-4.

Hoteit, I., Hoar, T., Gopalakrishnan, G., Collins, N., Anderson, J., Cornuelle, B., Köhl, A., Heimbach, P., 2013. A MITgcm/DART ensemble analysis and prediction system with application to the Gulf of Mexico. Dynamics of Atmospheres and Oceans 63, 1-23.

Hwang, C.-L., Masud, A. S. M., 1979. Multiple Objective Decision Making - Methods and Applications: A State-of-the-Art Survey. Springer Berlin Heidelberg, Berlin, Heidelberg.

Koay, T. B., Chitre, M., 2013. Energy-efficient path planning for fully propelled AUVs in congested coastal waters. In: OCEANS - Bergen, 2013 MTS/IEEE. pp. 1-9. 
Kularatne, D., Bhattacharya, S., Hsieh, M. A., 2016. Time and energy optimal path planning in general flows. In: Robotics: Science and Systems. pp. 1-10.

Lee, T., Kim, H., Chung, H., Bang, Y., Myung, H., 2015. Energy efficient path planning for a marine surface vehicle considering heading angle. Ocean Engineering 107, 118 131.

Liu, C., Zhao, Y., Gao, F., Liu, L., 2015. Three-dimensional path planning method for autonomous underwater vehicle based on modified firefly algorithm. Mathematical Problems in Engineering 2015, Article ID 561394.

Lolla, T., Lermusiaux, P. F. J., Ueckermann, M. P., Haley, P. J., 2014. Time-optimal path planning in dynamic flows using level set equations: theory and schemes. Ocean Dynamics 64 (10), 1373-1397.

Lolla, T., Ueckermann, M. P., Yigit, K., Haley, P. J., Lermusiaux, P. F. J., 2012. Path planning in time dependent flow fields using level set methods. In: Robotics and Automation (ICRA), 2012 IEEE International Conference on. pp. 166-173.

Niu, H., Ji, Z., Savvaris, A., Tsourdos, A., 2019. Energy efficient path planning for unmanned surface vehicle in spatially-temporally variant environment. Ocean Engineering, 106766.

Pêtrès, C., Pailhas, Y., Patron, P., Petillot, Y., Evans, J., Lane, D., 2007. Path planning for autonomous underwater vehicles. IEEE Transactions on Robotics 23 (2), 331-341.

Pêtrès, C., Pailhas, Y., Petillot, Y., Lane, D., 2005. Underwater path planing using fast marching algorithms. In: Oceans 2005-Europe. Vol. 2. IEEE, pp. 814-819.

Rao, D., Williams, S. B., 2009. Large-scale path planning for underwater gliders in ocean currents.

Rynne, P. F., von Ellenrieder, K. D., 2010. Development and preliminary experimental validation of a wind- and solar-powered autonomous surface vehicle. IEEE Journal of Oceanic Engineering 35 (4), 971-983.

Smith, R. N., Chao, Y., Li, P. P., Caron, D. A., Jones, B. H., Sukhatme, G. S., 2010. Planning and implementing trajectories for autonomous underwater vehicles to track 
evolving ocean processes based on predictions from a regional ocean model. The International Journal of Robotics Research 29 (12), 1475-1497.

Soulignac, M., 2011. Feasible and optimal path planning in strong current fields. IEEE Transactions on Robotics 27 (1), 89-98.

Soulignac, M., Taillibert, P., Rueher, M., 2008. Adapting the wavefront expansion in presence of strong currents. In: Robotics and Automation, 2008. ICRA 2008. IEEE International Conference on. pp. 1352-1358.

Soulignac, M., Taillibert, P., Rueher, M., 2009. Time-minimal path planning in dynamic current fields. In: Robotics and Automation, 2009. ICRA '09. IEEE International Conference on. pp. 2473-2479.

Stommel, H., 1989. The slocum mission. Oceanography 2.

Subramani, D. N., Haley, P. J., Lermusiaux, P. F. J., 2016. Energy-optimal path planning in the coastal ocean. Journal of Geophysical Research: Oceans 122 (5), 3981-4003.

Subramani, D. N., Lermusiaux, P. F., 2016. Energy-optimal path planning by stochastic dynamically orthogonal level-set optimization. Ocean Modelling 100, 57 - 77.

Subramani, D. N., Lolla, T., Haley, P. J., Lermusiaux, P. F. J., 2015. A stochastic optimization method for energy-based path planning. In: Ravela, S., Sandu, A. (Eds.), Dynamic Data-Driven Environmental Systems Science. Springer International Publishing, Cham, pp. 347-358.

Sun, F., Xu, W., Jin, L., Li, J., 2010. Path planning of autonomous underwater vehicles for optimal environmental sampling. In: OCEANS 2010 IEEE - Sydney. pp. 1-4.

Toye, H., Kortas, S., Zhan, P., Hoteit, I., 2018. A fault-tolerant hpc scheduler extension for large and operational ensemble data assimilation: Application to the red sea. Journal of Computational Science 27, $46-56$.

Toye, H., Zhan, P., Gopalakrishnan, G., Kartadikaria, A. R., Huang, H., Knio, O., Hoteit, I., 2017. Ensemble data assimilation in the Red Sea: Sensitivity to ensemble selection and atmospheric forcing. Ocean Dynamics 67 (7), 915-933. 
Wächter, A., Biegler, L. T., 2006. On the implementation of an interior-point filter linesearch algorithm for large-scale nonlinear programming. Mathematical Programming $106(1), 25-57$.

Wang, T., Le Maître, O. P., Hoteit, I., Knio, O. M., 2016. Path planning in uncertain flow fields using ensemble method. Ocean Dynamics 66 (10), 1231-1251.

Wang, T., Lima, R. M., Giraldi, L., Knio, O. M., 2019. Trajectory planning for autonomous underwater vehicles in the presence of obstacles and a nonlinear flow field using mixed integer nonlinear programming. Computers \& Operations Research 101, $55-75$.

Warren, C. W., 1990. A technique for autonomous underwater vehicle route planning. In: Autonomous Underwater Vehicle Technology, 1990. AUV '90., Proceedings of the (1990) Symposium on. pp. 201-205.

Yao, F., Hoteit, I., Feb 2016. A Modeling Study of Deep Water Renewal in the Red Sea. In: American Geophysical Union. Vol. 2016. pp. PO24A-2915.

Yao, F., Hoteit, I., Pratt, L., Bower, A., Köhl, A., Gopalakrishnan, G., Rivas, D., 2014a. Seasonal overturning circulation in the Red Sea: Part 2. Winter circulation. Journal of Geophysical Research: Oceans 119, 2263-2289.

Yao, F., Hoteit, I., Pratt, L., Bower, A., Zhai, P., Köhl, A., Gopalakrishnan, G., 2014b. Seasonal overturning circulation in the Red Sea: Part 1. Model validation and summer circulation. Journal of Geophysical Research: Oceans 119, 2238-2262.

Yilmaz, N. K., Evangelinos, C., Lermusiaux, P. F. J., Patrikalakis, N. M., 2008. Path planning of autonomous underwater vehicles for adaptive sampling using mixed integer linear programming. IEEE Journal of Oceanic Engineering 33 (4), 522-537.

Yuh, J., 2000. Design and control of autonomous underwater robots: A survey. Autonomous Robots 8 (1), 7-24. 
Table 1: Domain size and parameters for Case 1 and Case 2.

\begin{tabular}{|c|c|c|c|c|}
\hline \multicolumn{3}{|c|}{ Parameters } & Values & Units \\
\hline \multirow{2}{*}{\multicolumn{2}{|c|}{ Domain $\chi$}} & $\left(x_{\min }, z_{\min }\right)$ & $(0,0)$ & $m$ \\
\hline & & $\left(x_{\max }, z_{\max }\right)$ & $(100,50)$ & $m$ \\
\hline \multirow{2}{*}{\multicolumn{3}{|c|}{$\begin{array}{l}D_{\mathbf{x}_{0} \rightarrow \mathbf{x}^{f}} \\
\quad \Delta t\end{array}$}} & 80 & $m$ \\
\hline & & & 1 & $s$ \\
\hline \multirow{6}{*}{\multicolumn{2}{|c|}{ Case 1}} & $\mathbf{x}_{0}$ & $(10,50)$ & $m$ \\
\hline & & $\mathbf{x}^{f}$ & $(90,50)$ & $m$ \\
\hline & & $v_{\max }$ & 0.5 & $\mathrm{~m} / \mathrm{s}$ \\
\hline & & $a_{\max }$ & 0.5 & $\mathrm{~m} / \mathrm{s}^{2}$ \\
\hline & & $u_{x}(z, t)$ & 1 & $\mathrm{~m} / \mathrm{s}$ \\
\hline & & $T_{\max }$ & 160 & $s$ \\
\hline \multirow{12}{*}{ Case2 } & \multirow{6}{*}{ Instance I } & $\mathbf{x}_{0}$ & $(10,50)$ & $m$ \\
\hline & & $\mathbf{x}^{f}$ & $(90,50)$ & $m$ \\
\hline & & $v_{\max }$ & 1 & $\mathrm{~m} / \mathrm{s}$ \\
\hline & & $a_{\max }$ & 1 & $\mathrm{~m} / \mathrm{s}^{2}$ \\
\hline & & $u_{x}(z, t)$ & $\exp \left(-(z(t)-50)^{2} / 100\right)$ & $\mathrm{m} / \mathrm{s}$ \\
\hline & & $T_{\max }$ & 300 & $s$ \\
\hline & \multirow{6}{*}{ Instance II } & $\mathbf{x}_{0}$ & $(90,50)$ & $m$ \\
\hline & & $\mathbf{x}^{f}$ & $(10,50)$ & $m$ \\
\hline & & $v_{\max }$ & 1 & $\mathrm{~m} / \mathrm{s}$ \\
\hline & & $a_{\max }$ & 1 & $\mathrm{~m} / \mathrm{s}^{2}$ \\
\hline & & $u_{x}(z, t)$ & $\exp \left(-(z(t)-50)^{2} / 100\right)$ & $\mathrm{m} / \mathrm{s}$ \\
\hline & & $T_{\max }$ & 1500 & $s$ \\
\hline Maximum time set ${ }^{\dagger}$ & & Energy optimization step & $100 \mathrm{~s}$ & $s$ \\
\hline
\end{tabular}

$\dagger$ - Maximum time set per iteration in the solution procedure described in Section 3. 
Table 2: Domain size and parameters for Case 3 and Case 4.

\begin{tabular}{|c|c|c|c|c|}
\hline \multicolumn{3}{|c|}{ Parameters } & \multirow{2}{*}{$\begin{array}{l}\text { Values } \\
(1466090,117500,-900)\end{array}$} & \multirow{2}{*}{$\frac{\text { Units }}{m}$} \\
\hline & \multirow{2}{*}{ Domain $\chi$} & $\left(x_{\min }, z_{\min }\right)$ & & \\
\hline & & $\left(x_{\max }, z_{\max }\right)$ & $(1766880,248750,-2)$ & $m$ \\
\hline & & $D_{\mathbf{x}_{0} \rightarrow \mathbf{x}^{f}}$ & 234931.3 & $m$ \\
\hline & & $\Delta t$ & 3600 & $s$ \\
\hline & & $v_{\max }$ & 1 & $\mathrm{~m} / \mathrm{s}$ \\
\hline & & $a_{\max }$ & 1 & $\mathrm{~m} / \mathrm{s}^{2}$ \\
\hline & & $v_{\max _{z}}$ & 0.15 & $\mathrm{~m} / \mathrm{s}$ \\
\hline & & $a_{\max z}$ & 0.15 & $\mathrm{~m} / \mathrm{s}^{2}$ \\
\hline & & $u_{\max }$ & 1.53 & $\mathrm{~m} / \mathrm{s}$ \\
\hline & & $T_{\max }$ & 145 & $h$ \\
\hline \multirow{4}{*}{ Case 3} & \multirow{2}{*}{ Instance I } & $\mathbf{x}_{0}$ & $(1728207,213518)$ & $m$ \\
\hline & & $\mathbf{x}^{f}$ & $(1499340,160484)$ & $m$ \\
\hline & \multirow{2}{*}{ Instance II } & $\mathbf{x}_{0}$ & $(1499340,160484)$ & $m$ \\
\hline & & $\mathbf{x}^{f}$ & $(1728207,213518)$ & $m$ \\
\hline \multirow{4}{*}{ Case 4} & \multirow{2}{*}{ Instance I } & $\mathbf{x}_{0}$ & $(1728207,213518,-2)$ & $m$ \\
\hline & & $\mathbf{x}^{f}$ & $(1499340,160484,-2)$ & $m$ \\
\hline & \multirow{2}{*}{ Instance II } & $\mathbf{x}_{0}$ & $(1499340,160484,-2)$ & $m$ \\
\hline & & $\mathbf{x}^{f}$ & $(1728207,213518,-2)$ & $m$ \\
\hline Maximum time set ${ }^{\dagger}$ & & energy optimization step & 300 & $s$ \\
\hline
\end{tabular}

${ }^{\dagger}$ - Maximum time set per iteration in the solution procedure described in Section 3.

Table 3: Size of the models and computational results

\begin{tabular}{ccccc|cc|c}
\hline \hline & & \multicolumn{3}{c|}{ minimum time } & \multicolumn{2}{|c}{ minimum energy at $t_{\text {max }}$} & $\begin{array}{c}\text { average over } \\
\text { all problems }\end{array}$ \\
\cline { 3 - 8 } & & \# VAR & \# EQ & $T(\mathrm{~s})$ & \# VAR & \# EQ & $\overline{\mathrm{T}}(\mathrm{s})$ \\
\hline Case 1 & 751 & 747 & 30 & 4661 & 4657 & 156 \\
\hline \multirow{2}{*}{ Case 2 } & Instance I & 813 & 809 & 36 & 21013 & 21009 & 185 \\
& Instance II & 813 & 809 & 32 & 101513 & 101609 & 264 \\
\hline \multirow{2}{*}{ Case 3 } & Instance I & 762 & 872 & 795 & 1893 & 2177 & 258 \\
& Instance II & 762 & 872 & 890 & 1893 & 2177 & 289 \\
\hline \multirow{2}{*}{ Case 4 } & Instance I & 827 & 947 & 734 & 1971 & 2267 & 274 \\
& Instance II & 827 & 947 & 918 & 1971 & 2267 & 298 \\
\hline \hline
\end{tabular}

\#VAR: number of variables; \#EQ: number of equations; $T$ : wall clock time; $\overline{\mathrm{T}}$ : average wall clock time per energy consumption minimization step. Note that the number of equations and variables in Case 2 is due to the larger final time (cf. $T_{\max }$ in Table 1 ). 


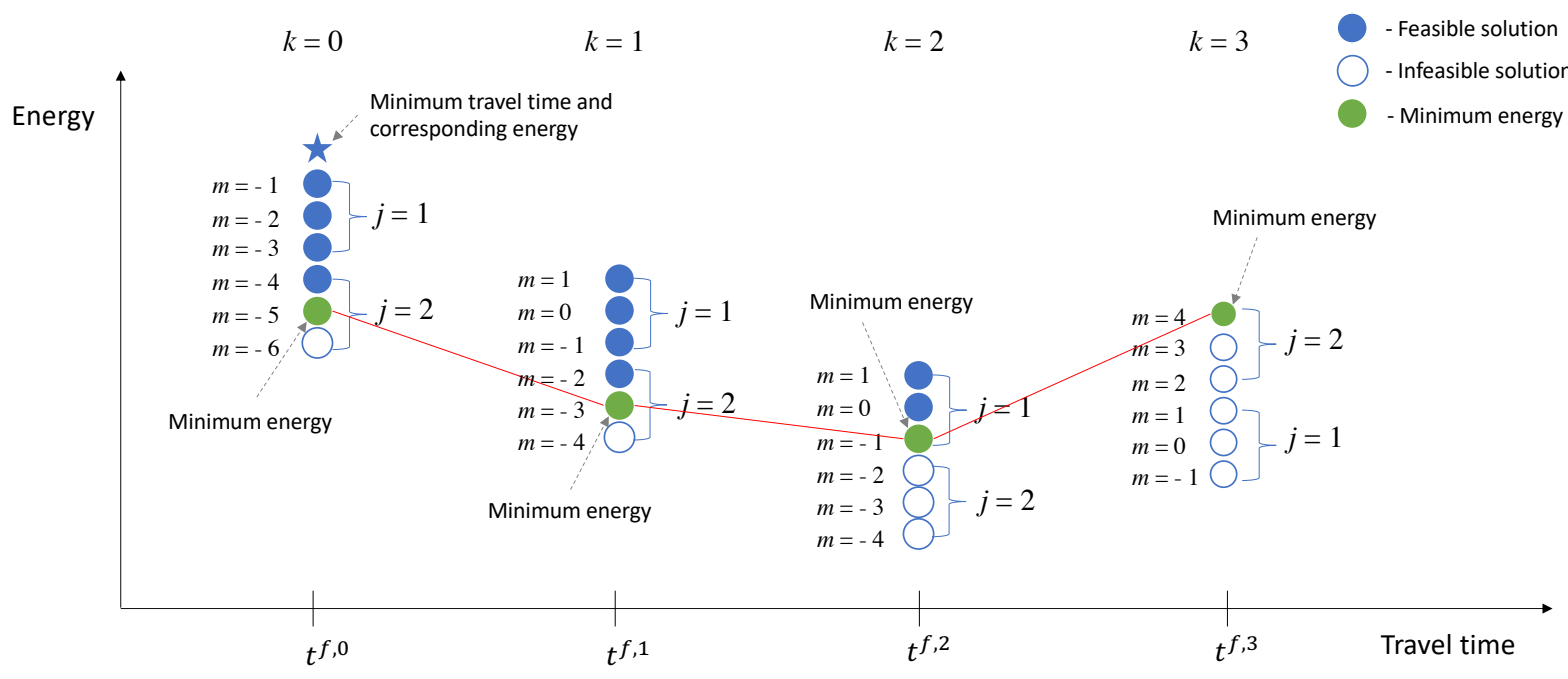

Figure 1: Illustrative example with $\bar{m}_{\max }=3$ that shows that for $t^{f, 0}$ only decreasing levels of energy are considered to solve Problem (7); the minimum energy is found with $m=-5$. For $t^{f, 1}$, the procedure starts with $\mathcal{M}^{1} \mid m \in\{-1 ; 0,1\}$ to solve Problem (7) and then it moves to $\mathcal{M}^{2} \mid m \in\{-2 ;-3,-4\}$; the minimum energy is found with $m=-3$. For $t^{f, 2}$, the procedure starts with $\mathcal{M}^{1} \mid m \in\{-1 ; 0,1\}$ to solve Problem (7) and then it moves to $\mathcal{M}^{2} \mid m \in\{-2 ;-3,-4\}$; the minimum energy is found with $m=-1$, because no feasible solution was found in $\mathcal{M}^{2}$. For $t^{f, 3}$, the procedure starts with $\mathcal{M}^{1} \mid m \in\{-1 ; 0,1\}$ to solve Problem (7) and then it moves to $\mathcal{M}^{2} \mid m \in\{2 ; 3,4\}$; the minimum energy is found with $m=4$. 


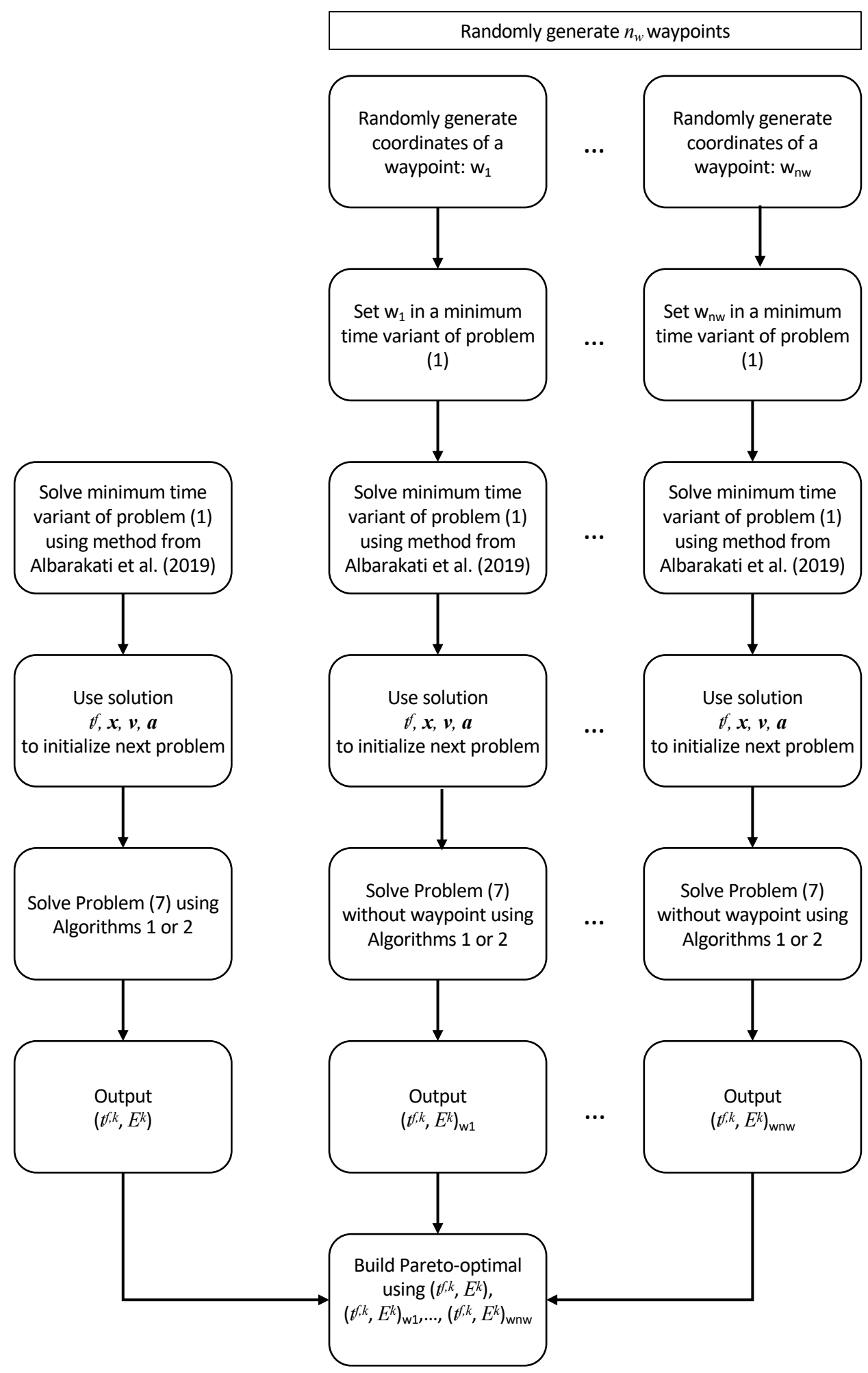

Figure 2: Diversification strategy to find additional Pareto-optimal solutions by using waypoints to find minimum time solutions. Note that the waypoints are not considered to solve Problem (7). 


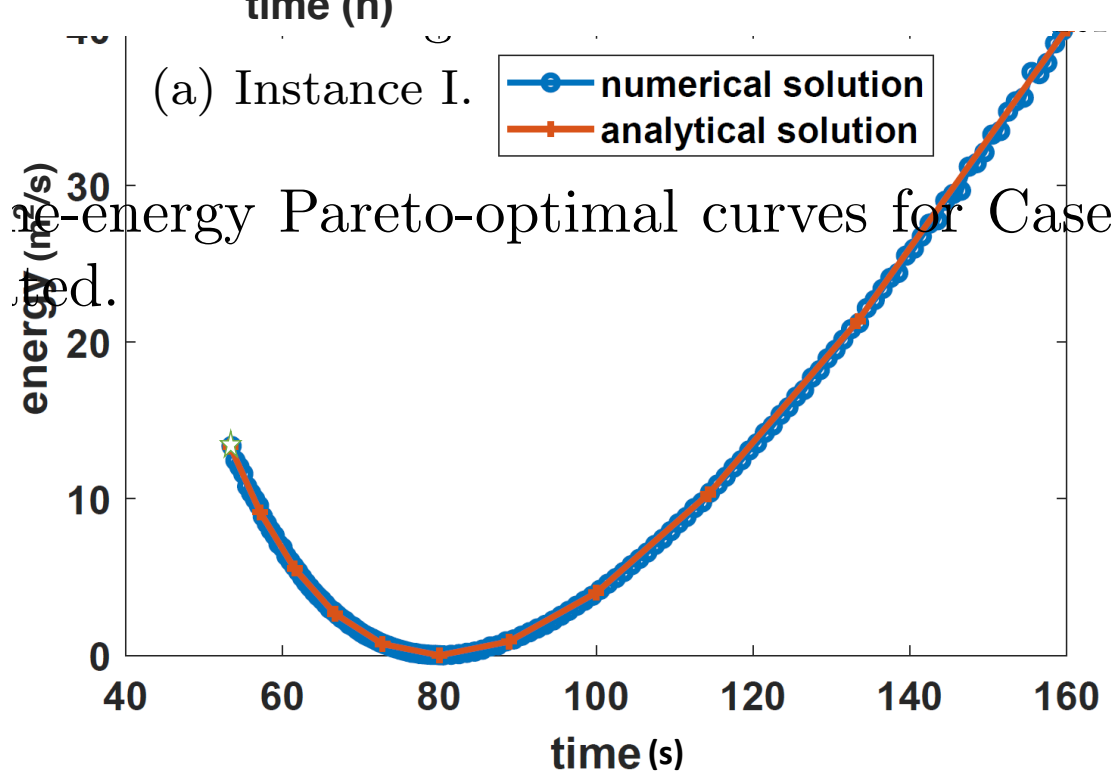

Figure 3: Time-energy Pareto-optimal curve plot for case 1. Plotted are numerical and analytical solutions

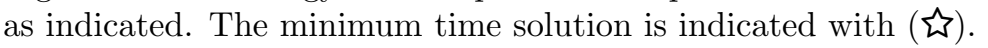




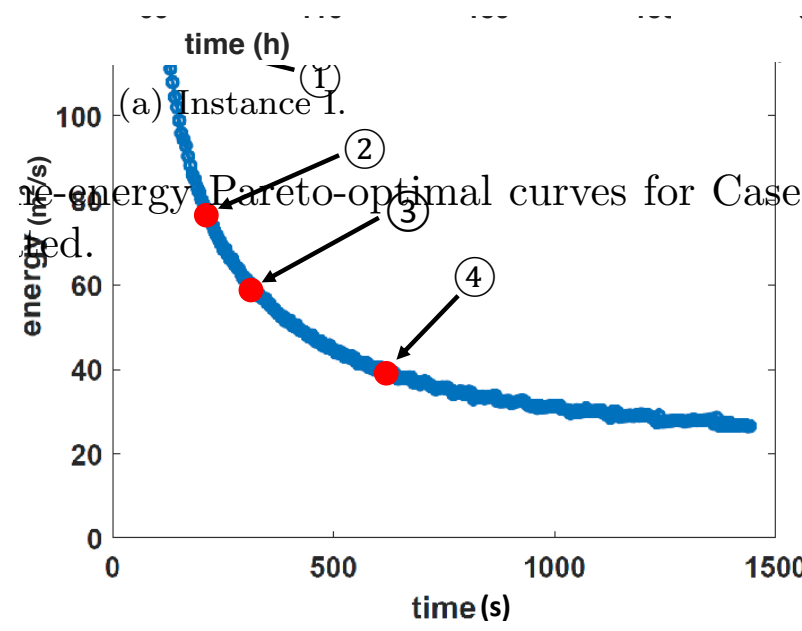

(a) Instance I.

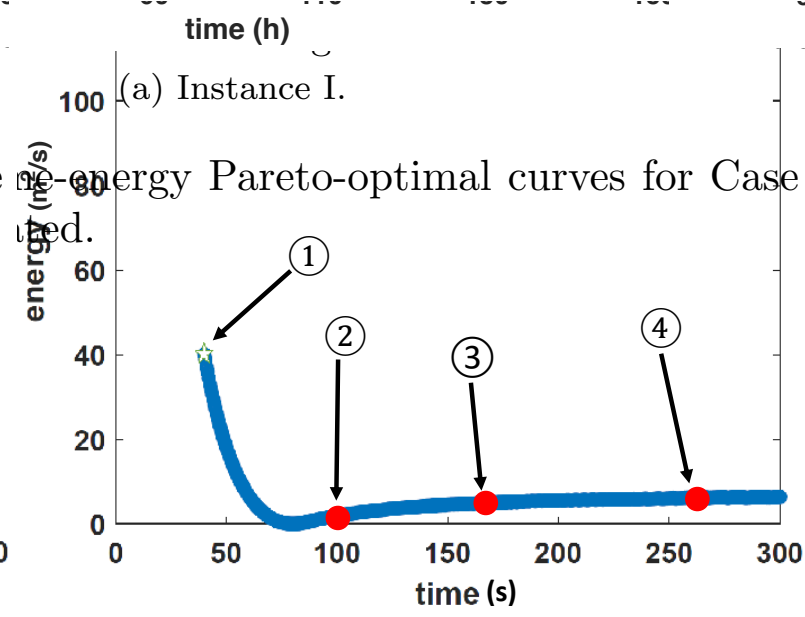

(b) Instance II.

Figure 4: Time-energy Pareto-optimal curves for Case2. The minimum time solutions are indicated with (今).

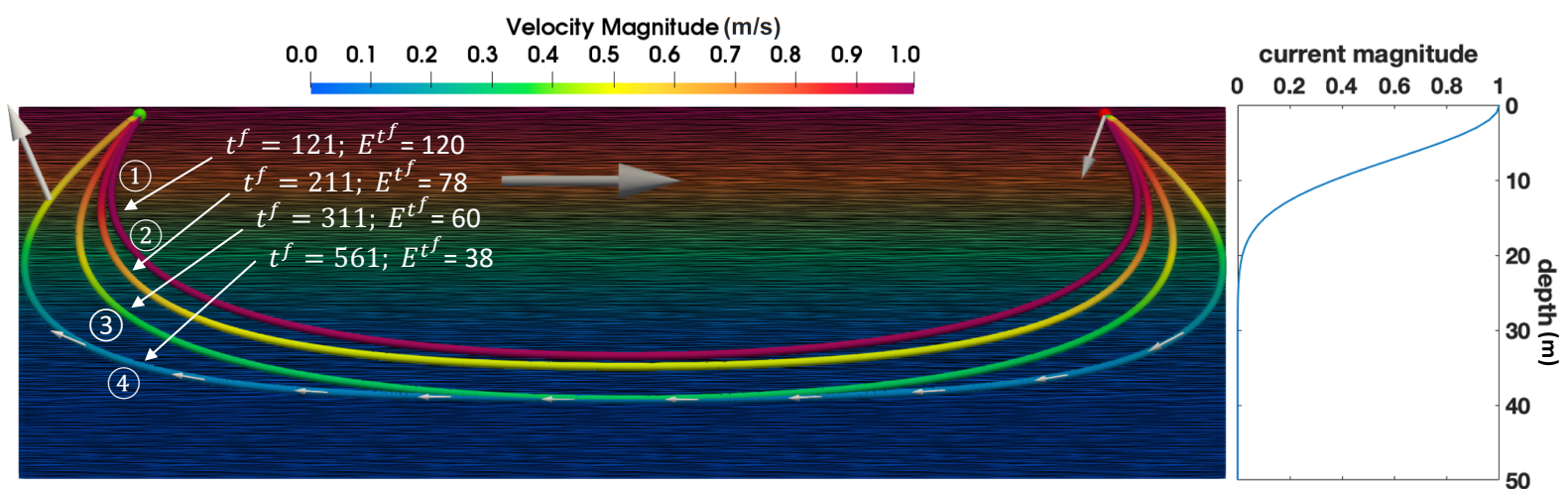

Figure 5: Case 2; Instance I. Left: Optimal path in the $x-z$ plane for different values of travel time and energy. The color contours depict the current velocity, and the central large gray arrow shows the direction of the current. The starting point is indicated by a red circle $(\bigcirc)$ and the target destination is specified in a green circle $(\bigcirc)$. The number on each path corresponds to the time-energy solution on the Pareto-optimal curve in Figure 4a. Right: vertical profile for the current velocity.

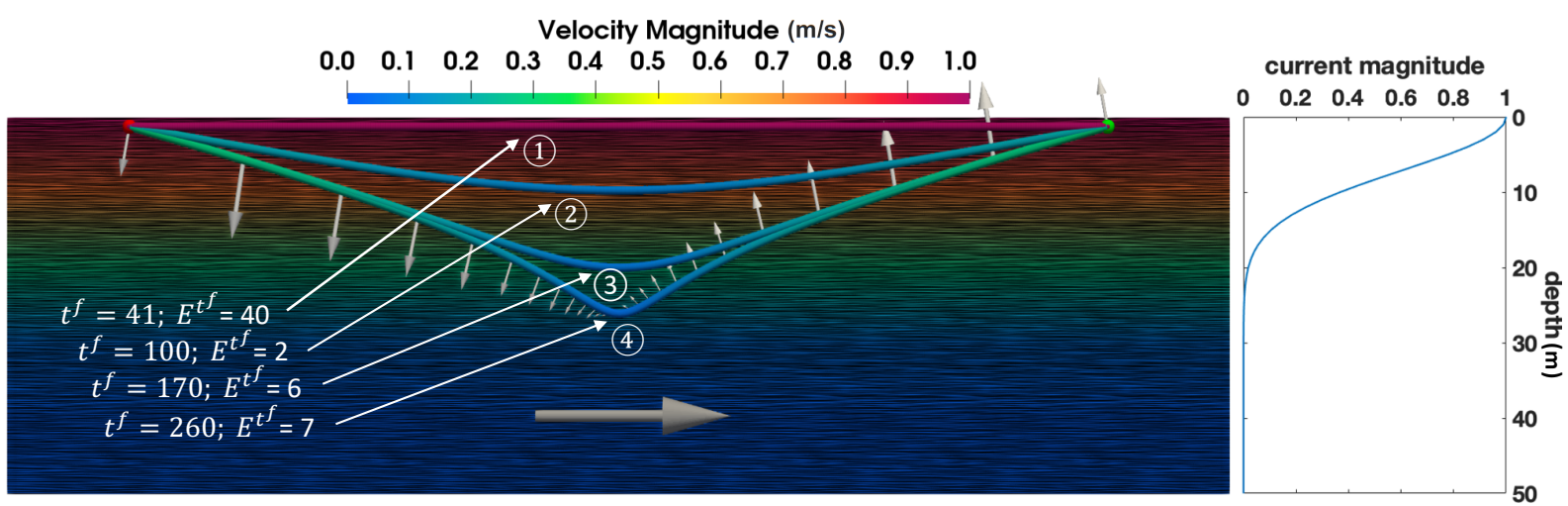

Figure 6: Case 2; Instance II. Left: Optimal path in the $x$ - $z$ plane for different values of travel time and energy. The color contours depict the current velocity, and the central large gray arrow shows the direction of the current. The starting point is indicated by a red circle $(\bigcirc)$ and the target destination is specified in a green circle $(\bigcirc)$. The number on each path corresponds to the time-energy solution on the Pareto-optimal curve in Figure 4b. Right: vertical profile for the current velocity. 


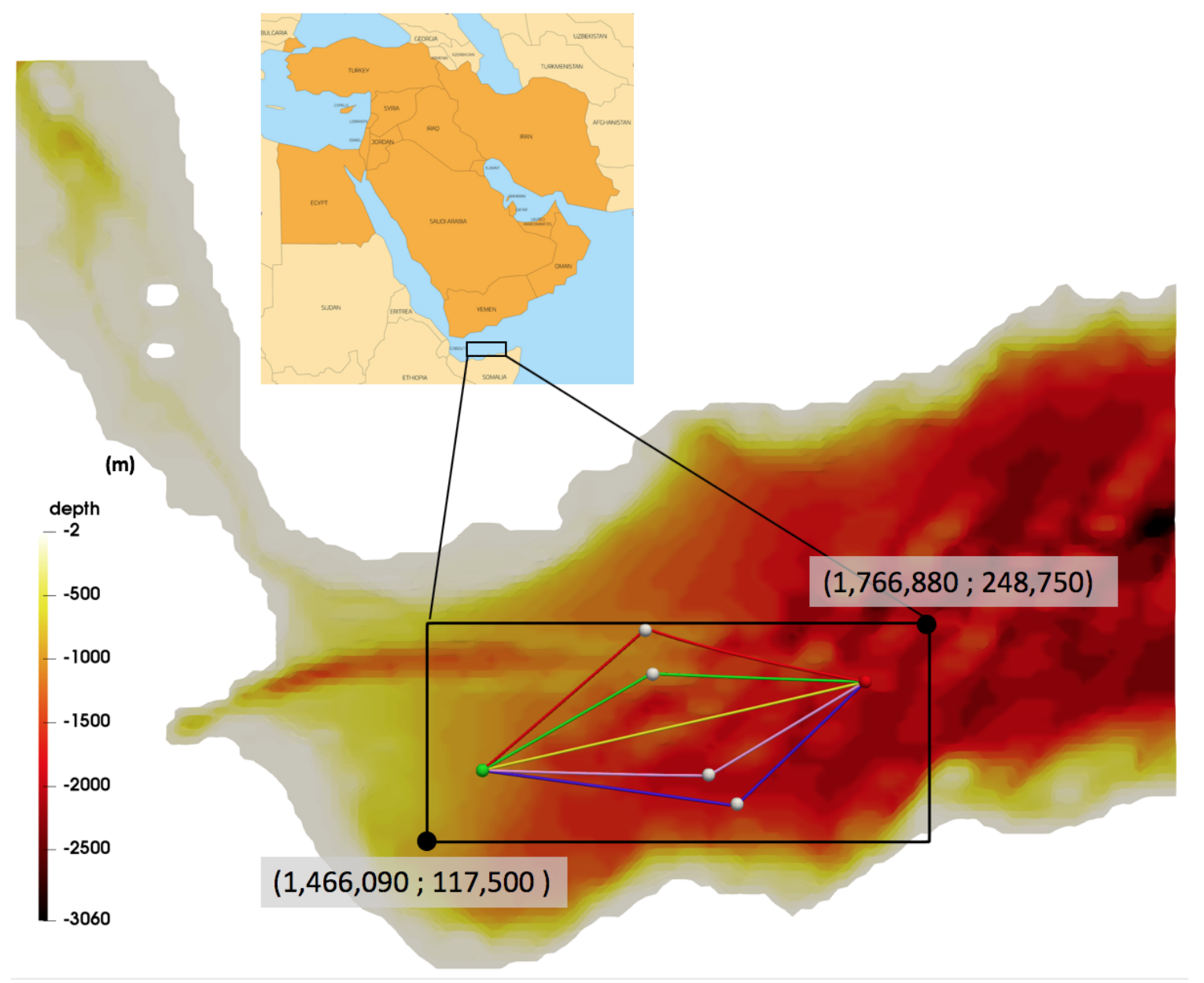

Figure 7: Gulf of Aden region. The rectangle indicates the problem domain (coordinates are in meters from origin of simulation domain) for Case 3 and Case 4, and the color contours indicate bathymetry. The red (O) and green (O) circles indicate the starting and end points for Instance I. (This is reversed in Instance II). The straight yellow curve is the no-current initialization without waypoint. The remaining curves are no-current initializations corresponding to different waypoints $(\bigcirc)$. 


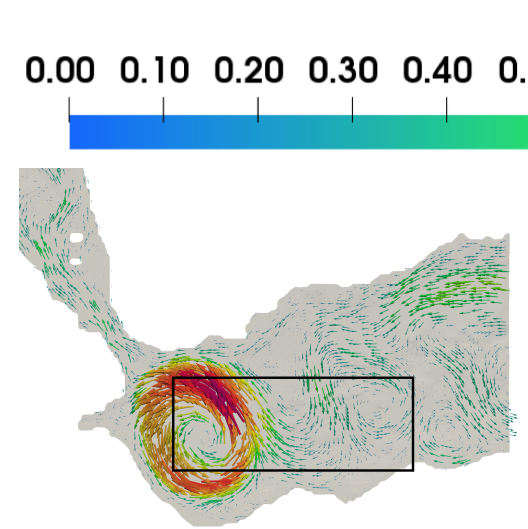

(a) Time $=0$ days; Depth $=-2 \mathrm{~m}$

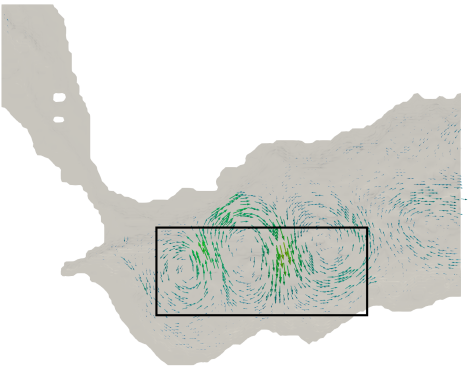

(d) Time $=0$ days; Depth $=-400 \mathrm{~m}$

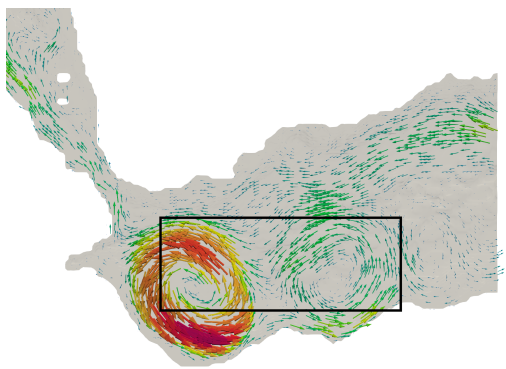

(b) Time $=3$ days; Depth $=-2 \mathrm{~m}$

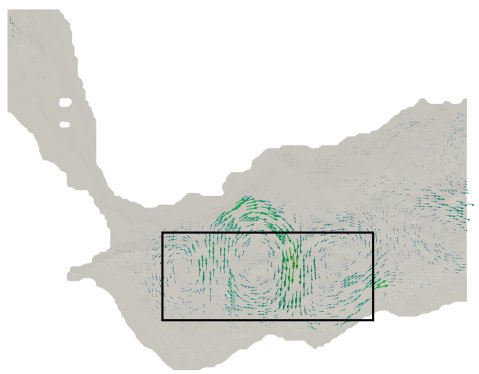

(e) Time $=3$ days; Depth $=-400 \mathrm{~m}$

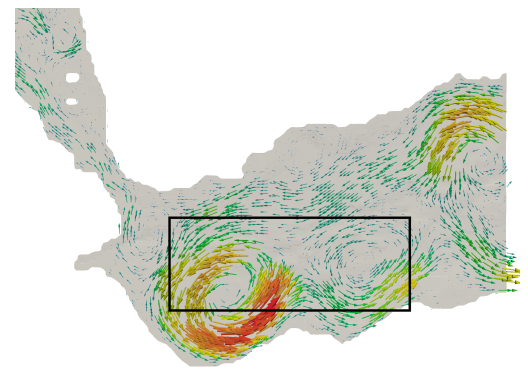

(c) Time $=6$ days; Depth $=-2 \mathrm{~m}$

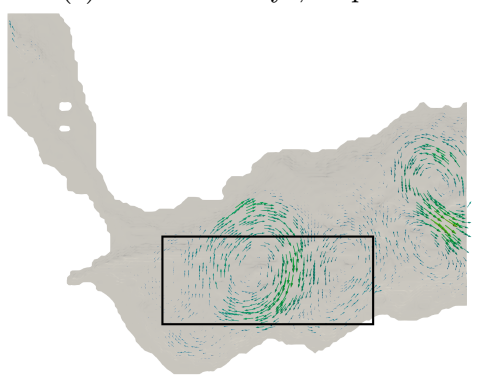

(f) Time $=6$ days; Depth $=-400 \mathrm{~m}$

Figure 8: Snapshots of the current field at different times and depths, as indicated (days counted from 19 January 2006). Arrows depict current velocity, colors indicate surface current magnitude, the rectangle indicates the problem domain (cf. Figure 7). 


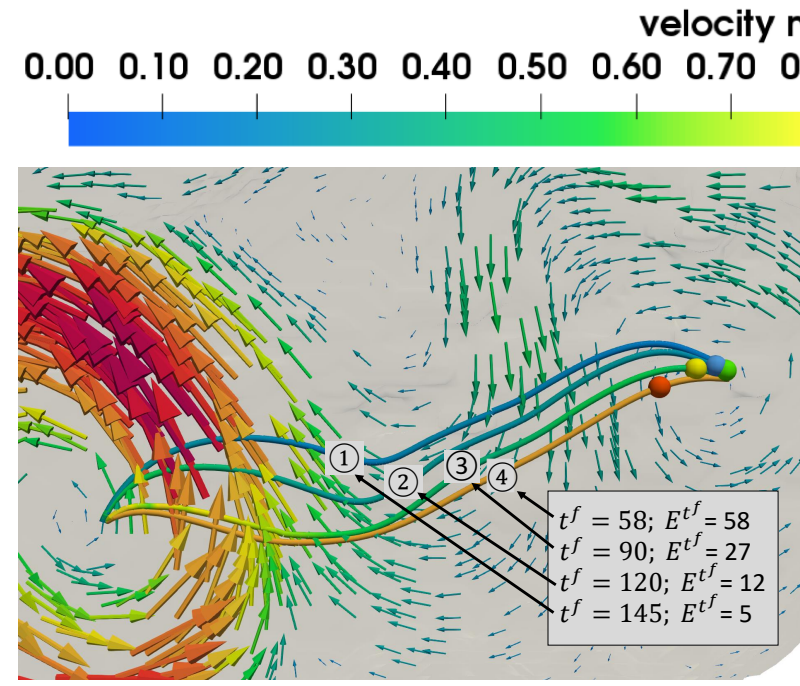

(a) Time $=8 \mathrm{~h}$.

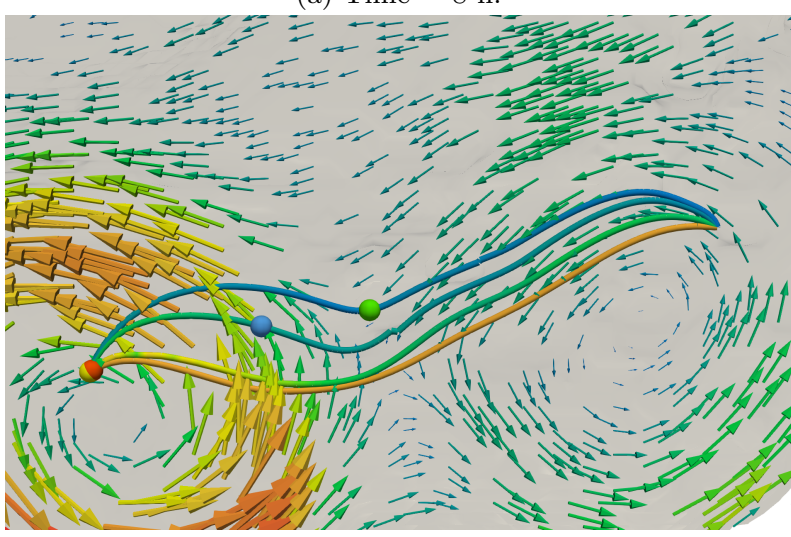

(c) Time $=98 \mathrm{~h}$.
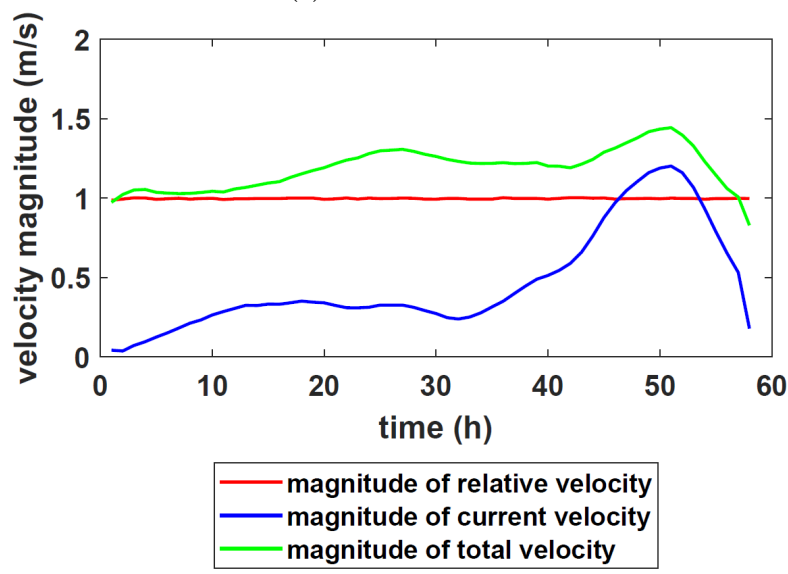

(e) Minimum time solution (labeled (1) in Figure 9a).

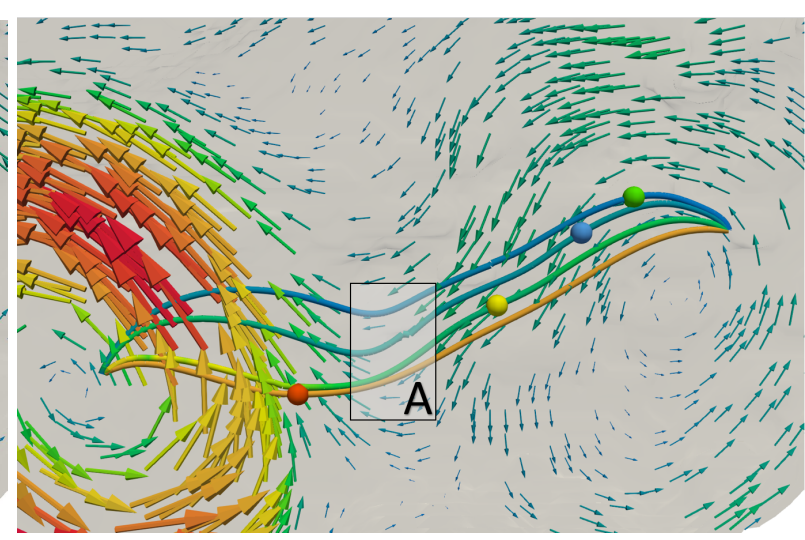

(b) Time $=42 \mathrm{~h}$.

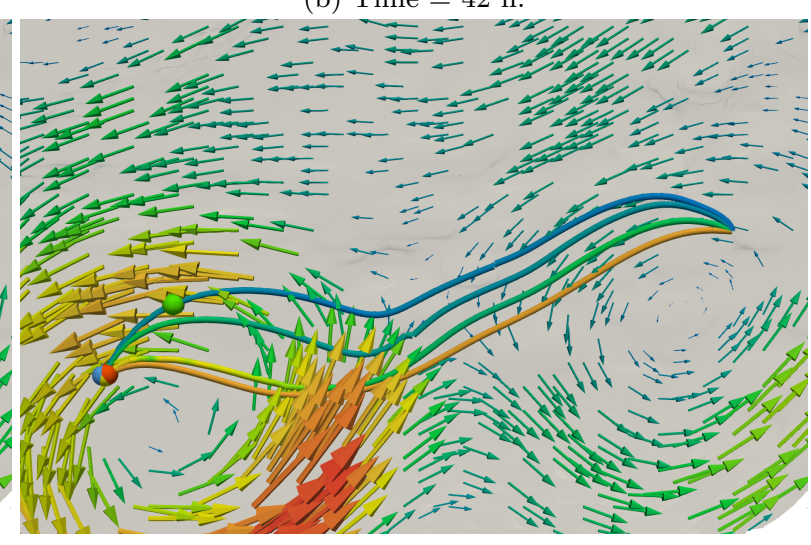

(d) Time $=134 \mathrm{~h}$.

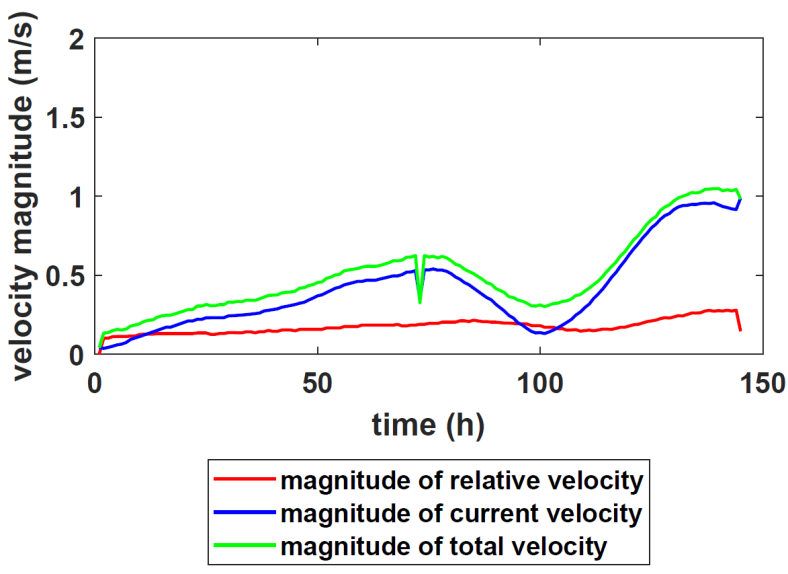

(f) Minimum energy solution (labeled (4) in Figure 9a).

Figure 9: Case 3 Instance I. (a) - (d) - instantaneous distributions of the surface current at different times and paths with different travel times, as indicated (hours counted from 19 January 2006). (e) - (f) - velocity profiles of the AUV for the minimum time and minimum energy solutions. 
velocity magnitude $(\mathrm{m} / \mathrm{s})$

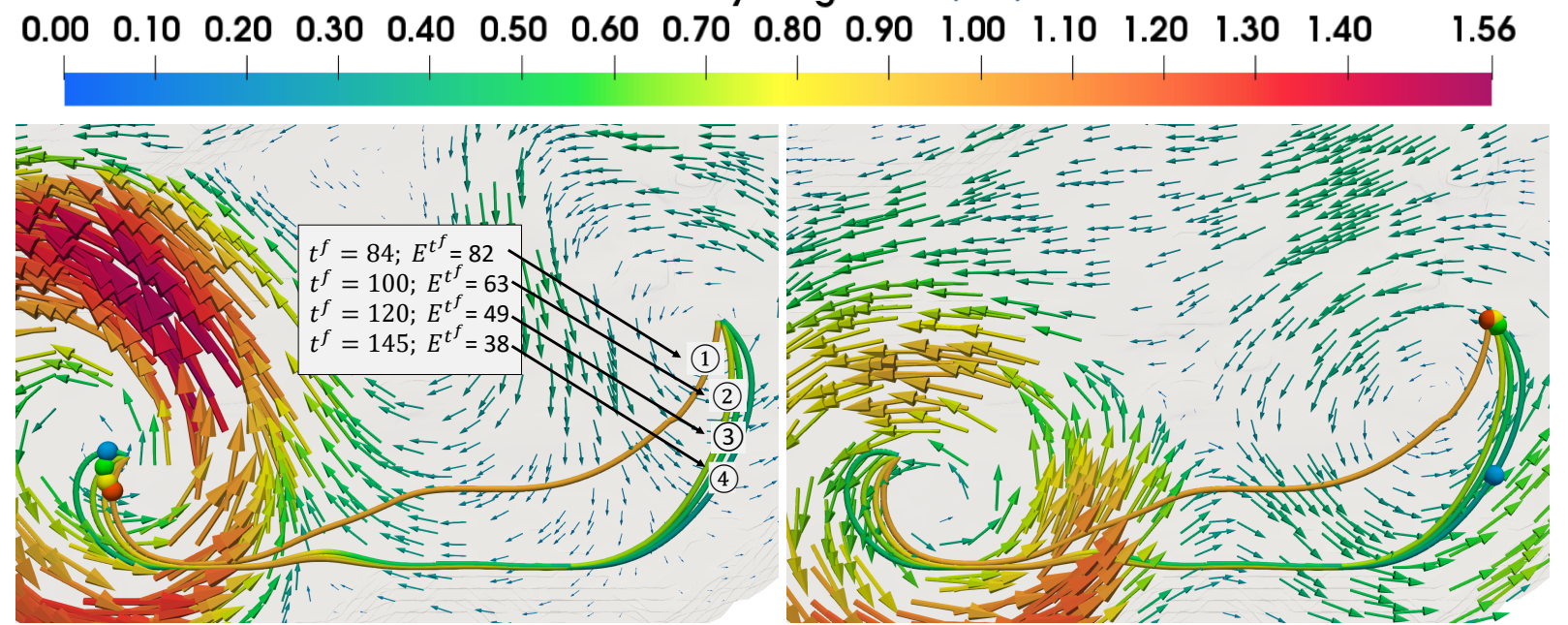

(a) Time $=5 \mathrm{~h}$.

(b) Time $=117 \mathrm{~h}$.
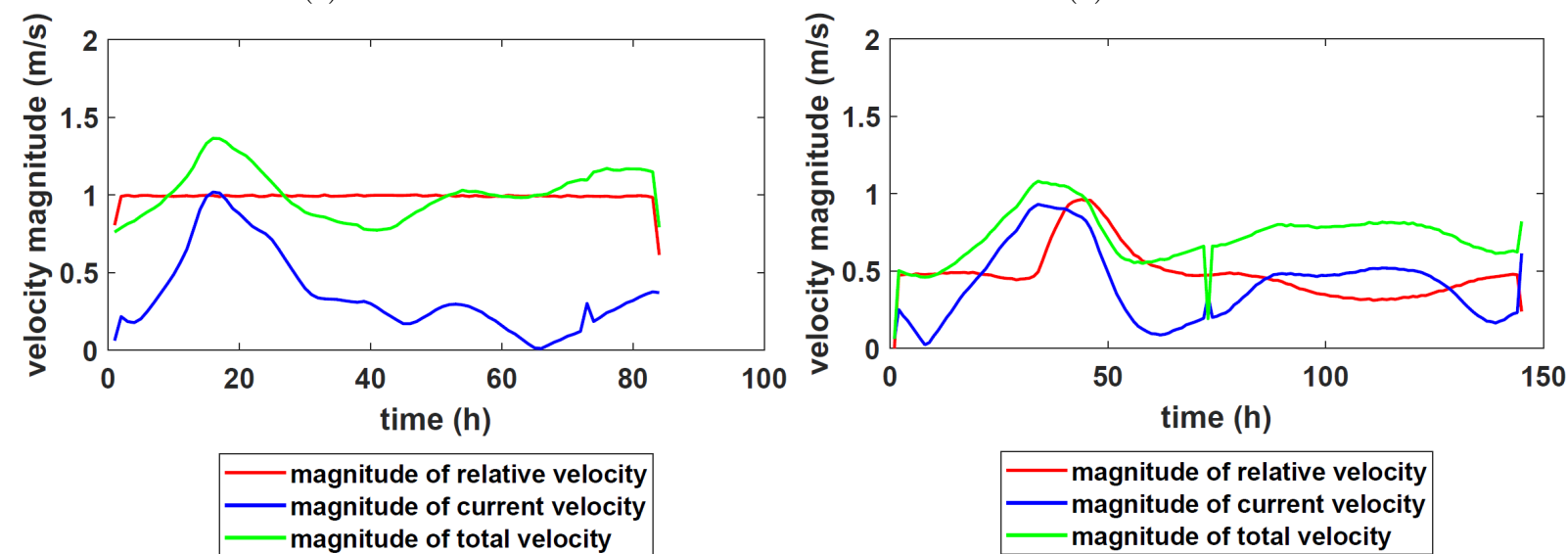

(c) minimum time solution (labeled (1) in Figure 10a).

(d) minimum energy solution (labeled (4) in Figure 10a).

Figure 10: Case 3 Instance II. (a) - (b) - instantaneous distributions of the surface current at different times and paths with different travel times, as indicated (hours counted from 19 January 2006). (c) - (d) - velocity profiles of the AUV for the minimum time and minimum energy solutions. 


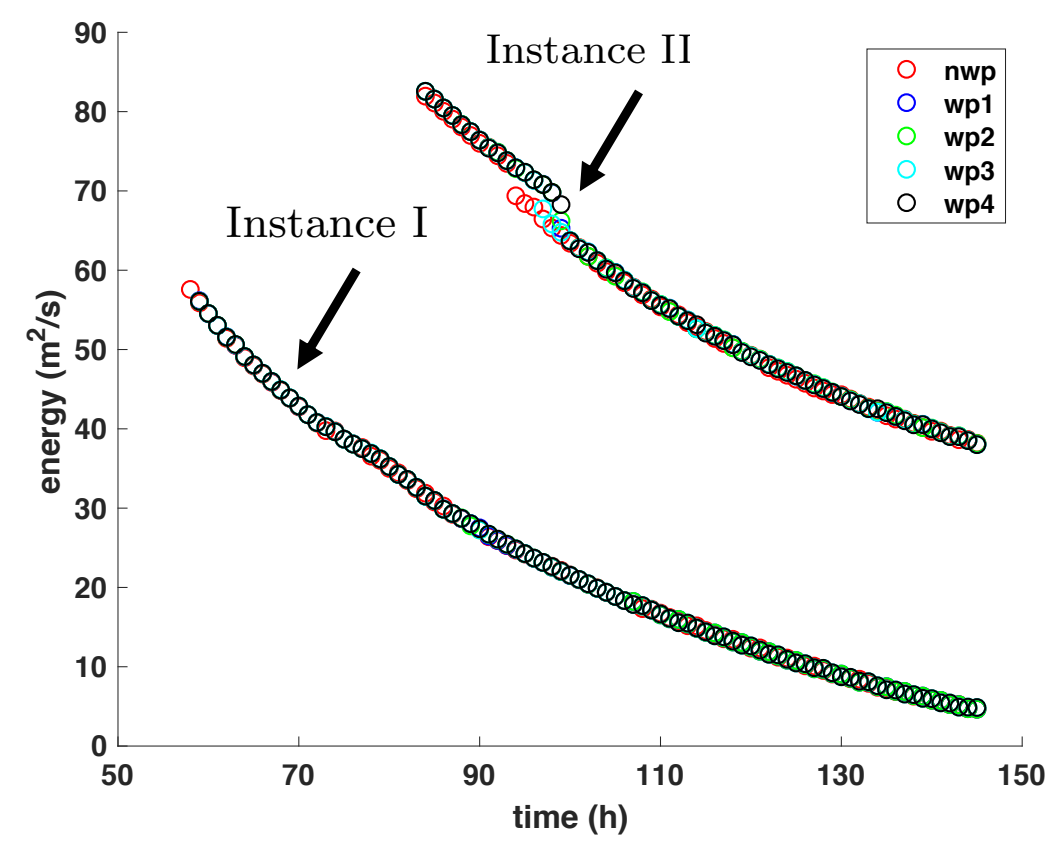

Figure 11: Time-energy Pareto-optimal curves for Case 3. Curves are generated for different initializations, as indicated.

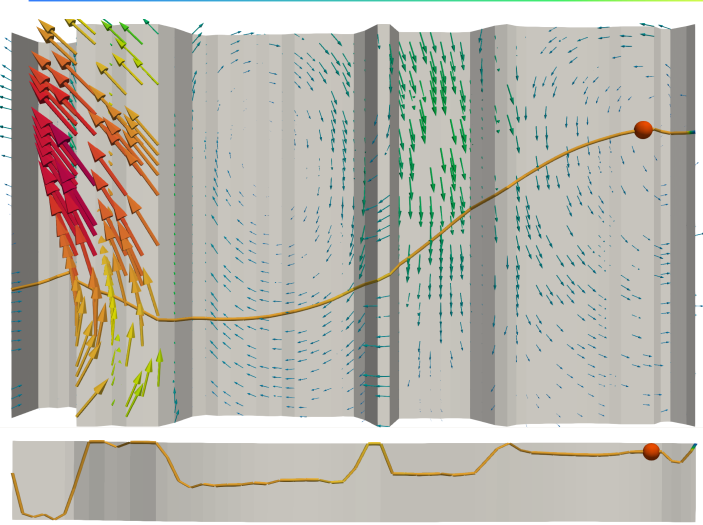

(a) Time $=4 \mathrm{~h}$.

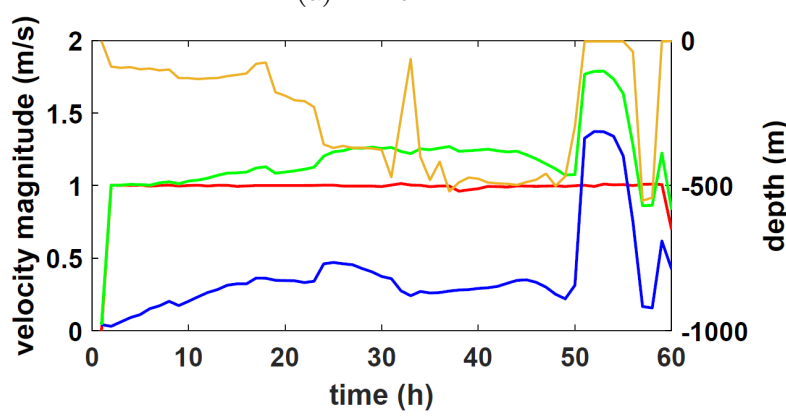

(c) Velocity and depth profile.

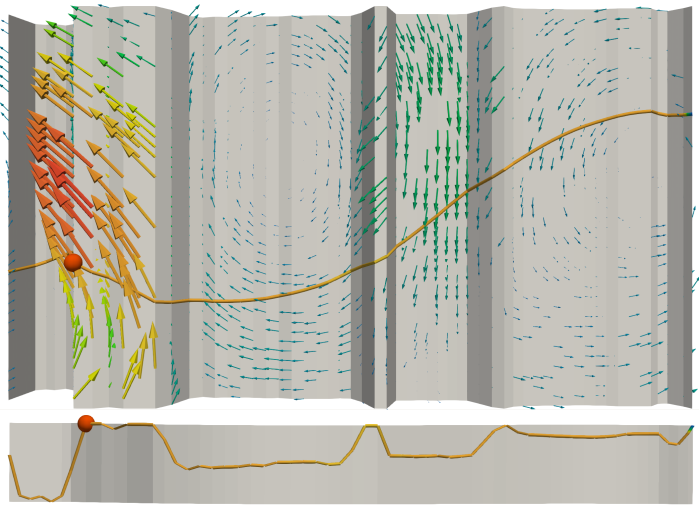

(b) Time $=51 \mathrm{~h}$.

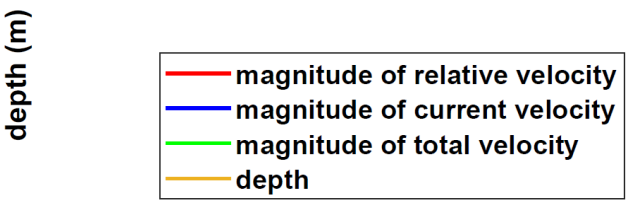

Figure 12: Minimum time solution for Case 4, Instance I. (a) - (b) - Each subfigure shows two views: 1) a top view of the vehicle path over instantaneous distributions of the current at the trajectory depth; and 2) a side view of the path. (c) - Velocity profile along the vehicle's path. The path of the vehicle as the flow are color coded with their velocity magnitudes. Depth has been scaled by 30 . Hours counted from 19 January 2006. 


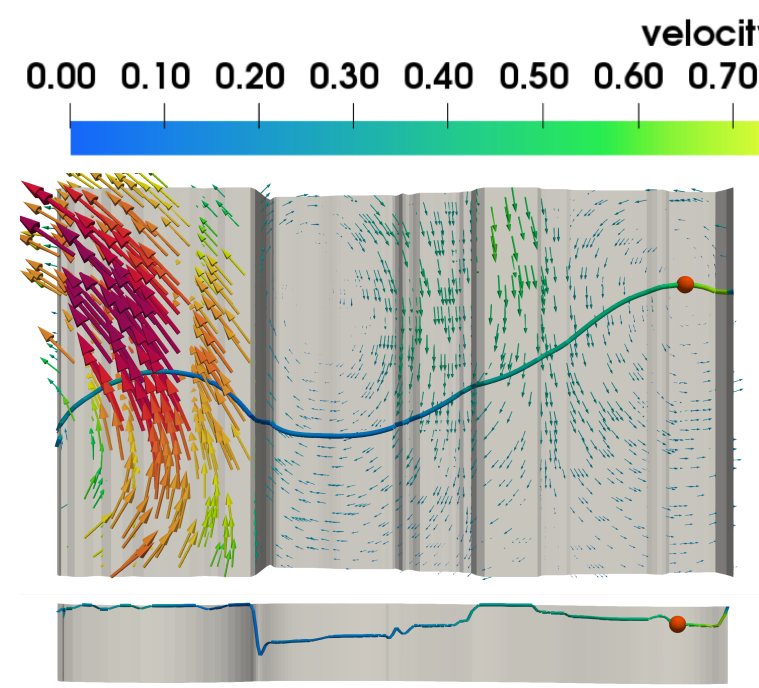

(a) Time $=8 \mathrm{~h}$.

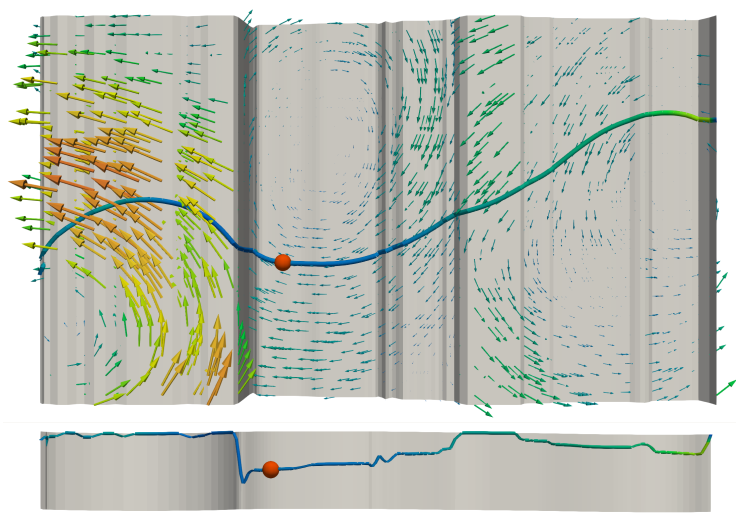

(c) Time $=98 \mathrm{~h}$.

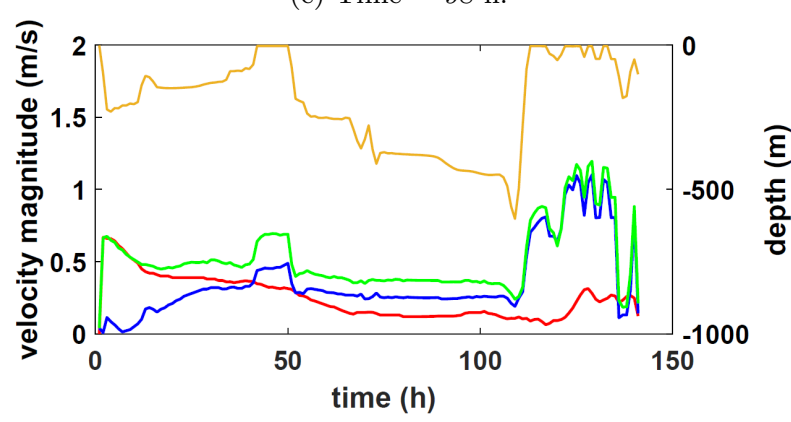

(e) Velocity and depth profile.

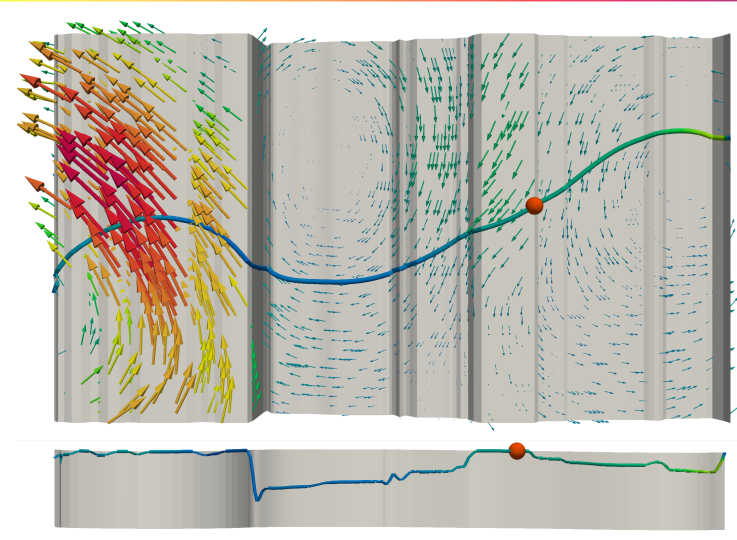

(b) Time $=42 \mathrm{~h}$.

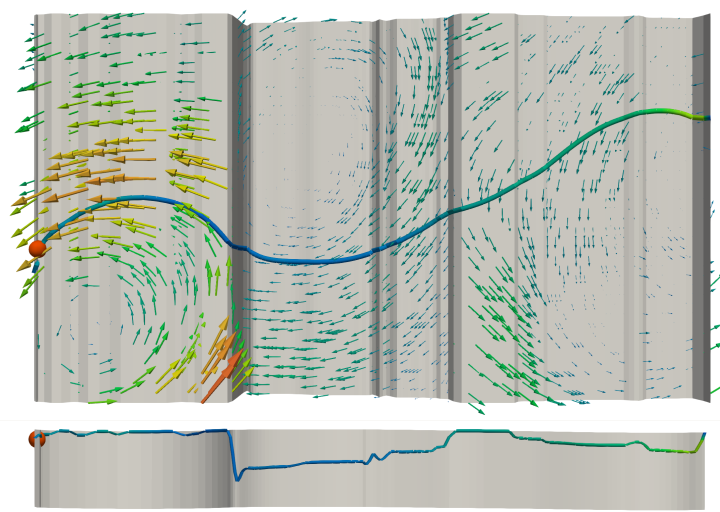

(d) Time $=134 \mathrm{~h}$. 


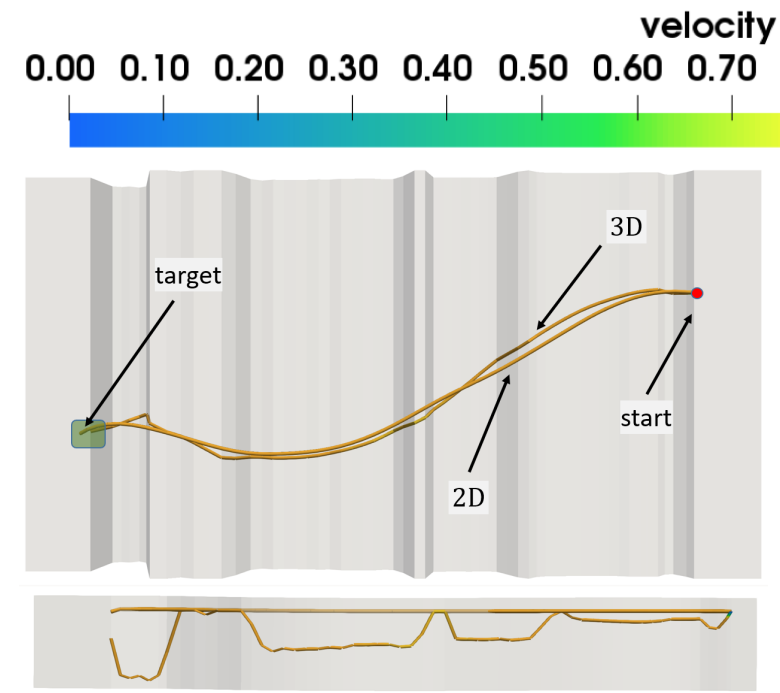

(a) Instance I - minimum time

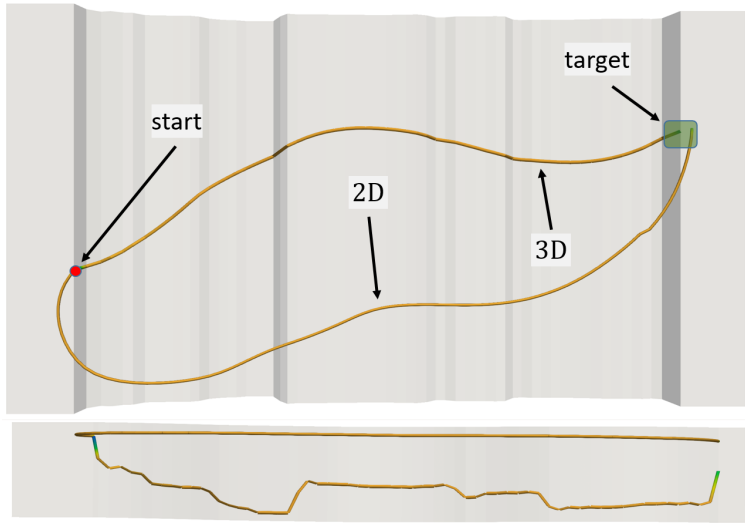

(c) Instance II - minimum time

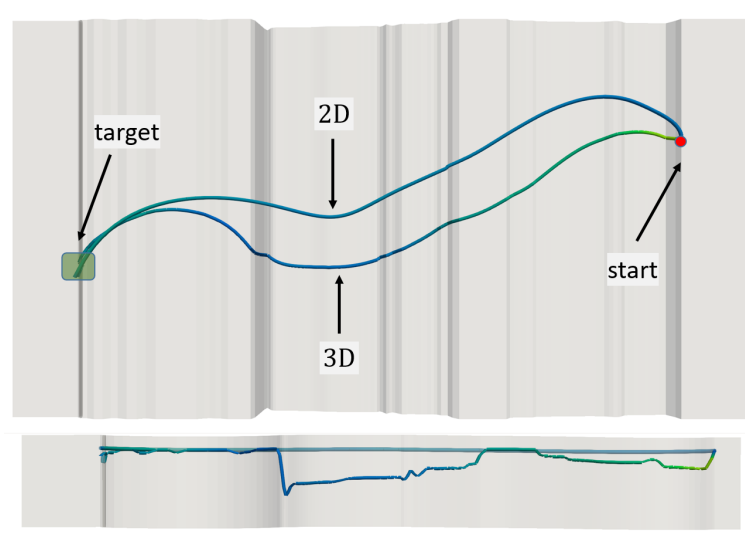

(b) Instance I - minimum energy

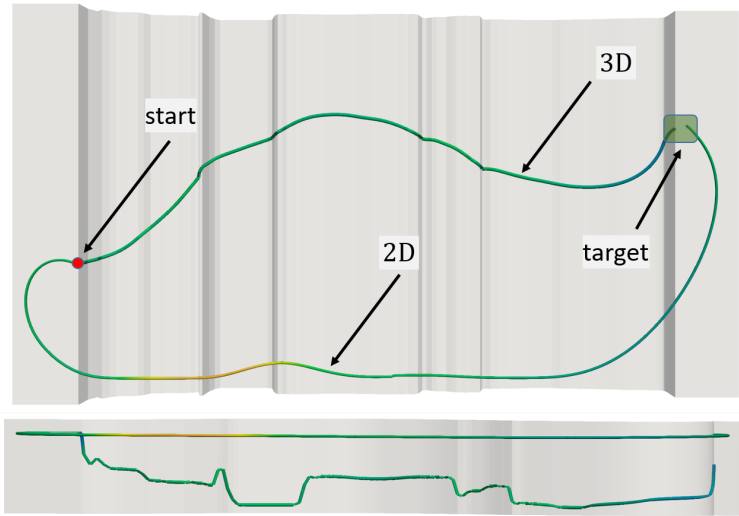

(d) Instance II - minimum energy

Figure 14: Optimal paths for Case 3 and Case 4, colored using the relative velocity of the AUV, as indicated. Each subfigure shows top and vertical views. 
velocity magnitude $(\mathrm{m} / \mathrm{s})$

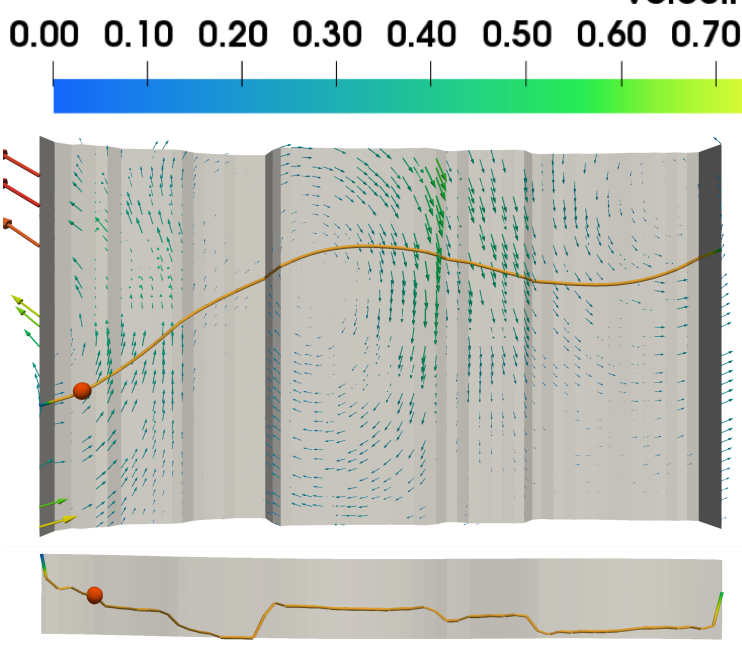

(a) Time $=4 \mathrm{~h}$.

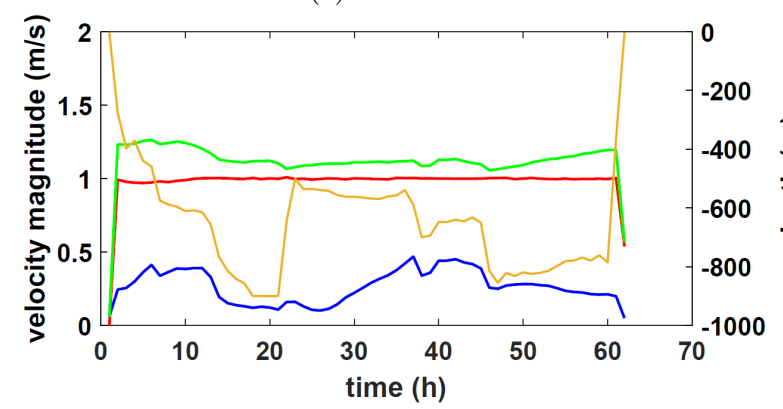

(c) Velocity and depth profile.

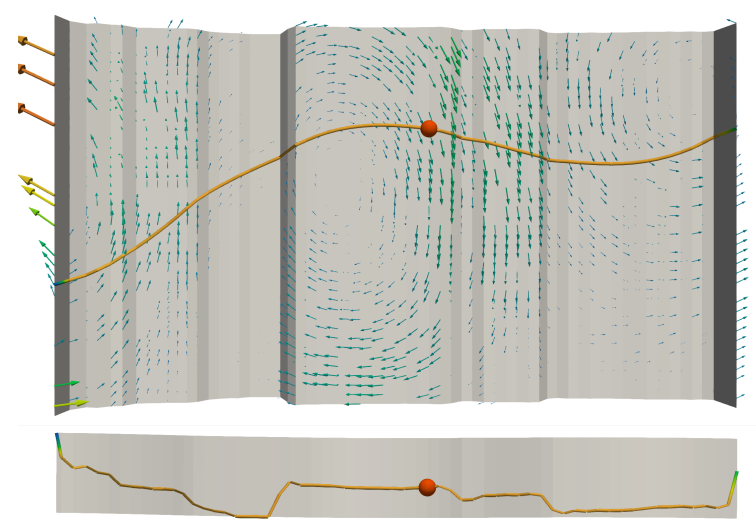

(b) Time $=34 \mathrm{~h}$.

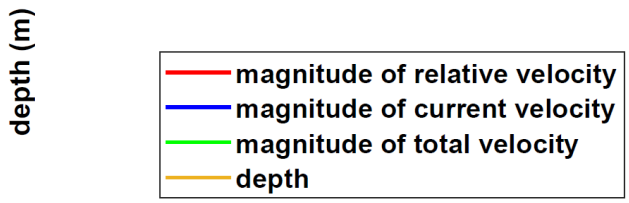

Figure 15: Minimum time solution for Case 4, Instance II. (a) -(b) - Each subfigure shows two views: 1) a top view of the vehicle path over instantaneous distributions of the current at the trajectory depth; and 2) a side view of the path. (c)- Velocity profile along the vehicle's path. The path of the vehicle as the flow are color coded with their velocity magnitudes. Depth has been scaled by 30. Hours counted from 19 January 2006. 
velocity magnitude $(\mathrm{m} / \mathrm{s})$

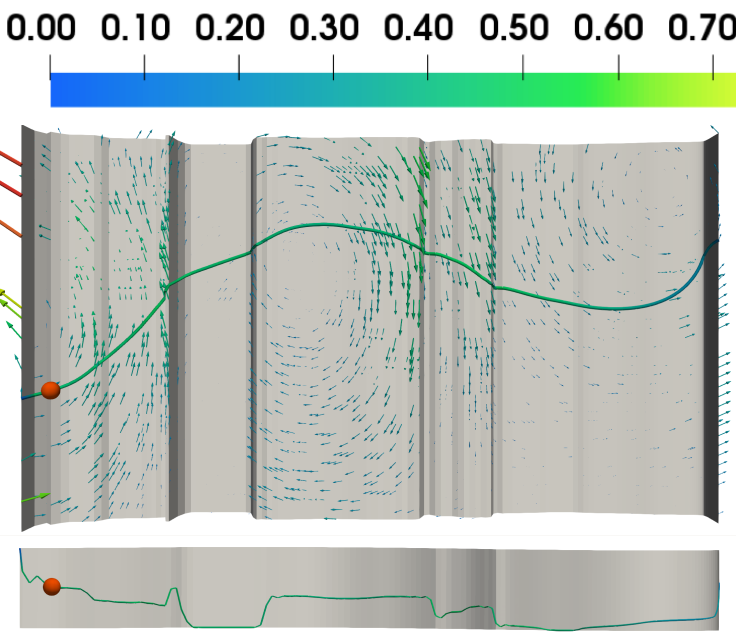

(a) Time $=4 \mathrm{~h}$.

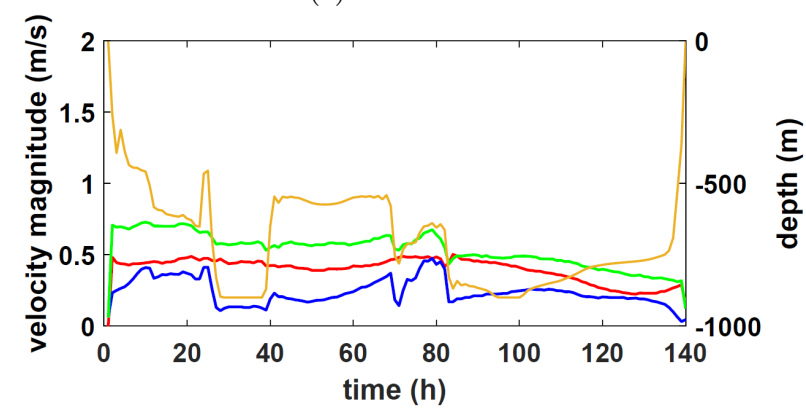

(c) Velocity and depth profile.

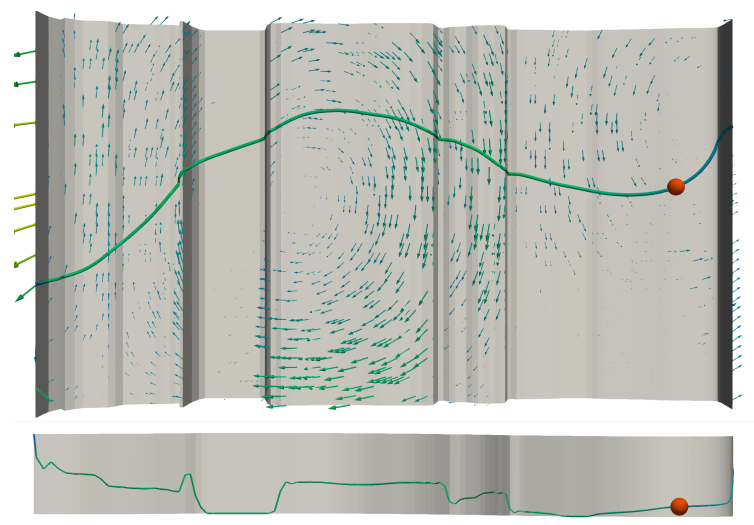

(b) Time $=118 \mathrm{~h}$.

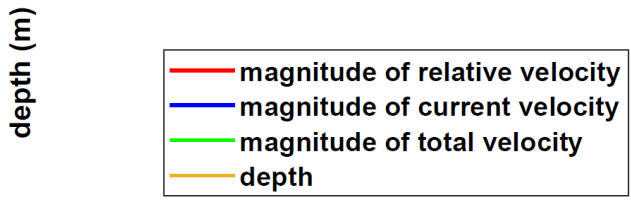

Figure 16: Minimum energy solution for Case 4, Instance II. (a) - (b) - Each subfigure shows two views: 1) a top view of the vehicle path over instantaneous distributions of the current at the trajectory depth; and 2) a side view of the path. (c)- Velocity profile along the vehicle's path. The path of the vehicle as the flow are color coded with their velocity magnitudes. Depth has been scaled by 30. Hours counted from 19 January 2006.

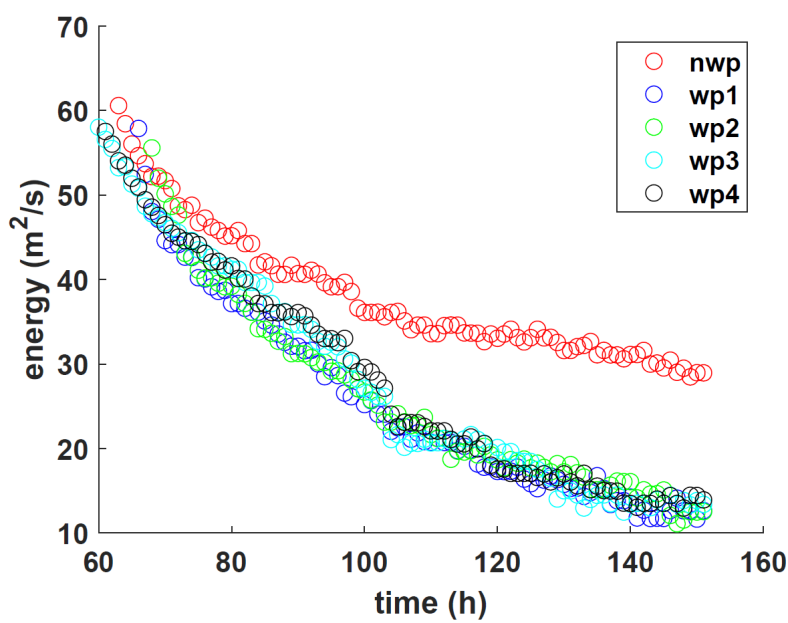

(a) Instance I.

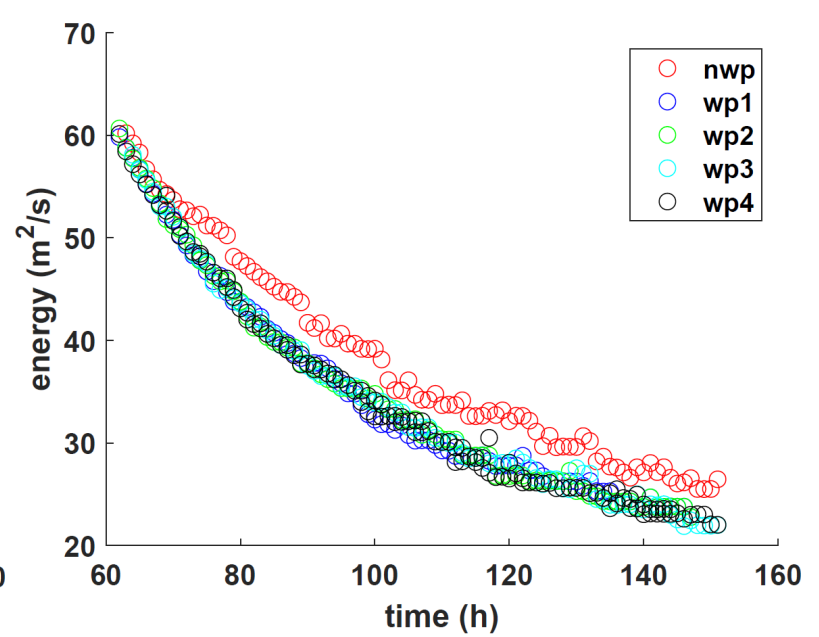

(b) Instance II.

Figure 17: Time-energy Pareto-optimal curves for Case 4. Curves are generated for different initializations, as indicated (hours counted from 19 January 2006). 


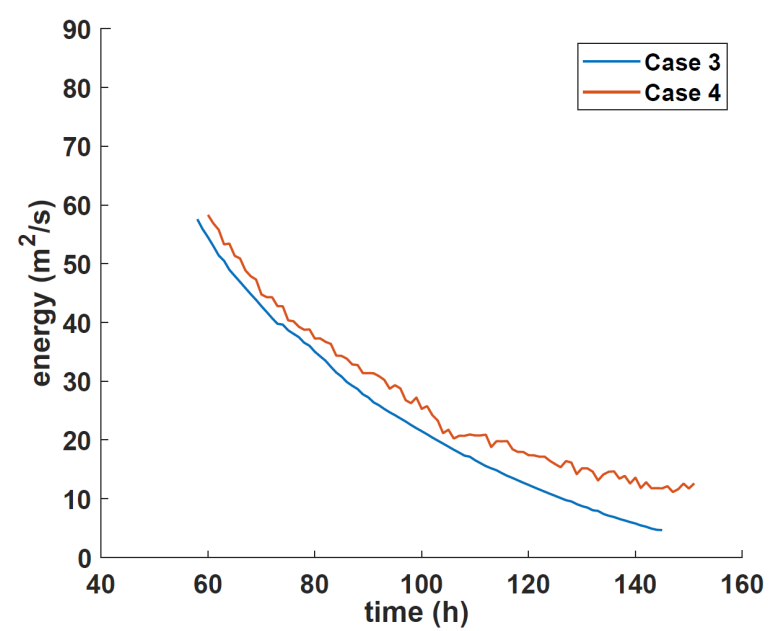

(a) Instance I.

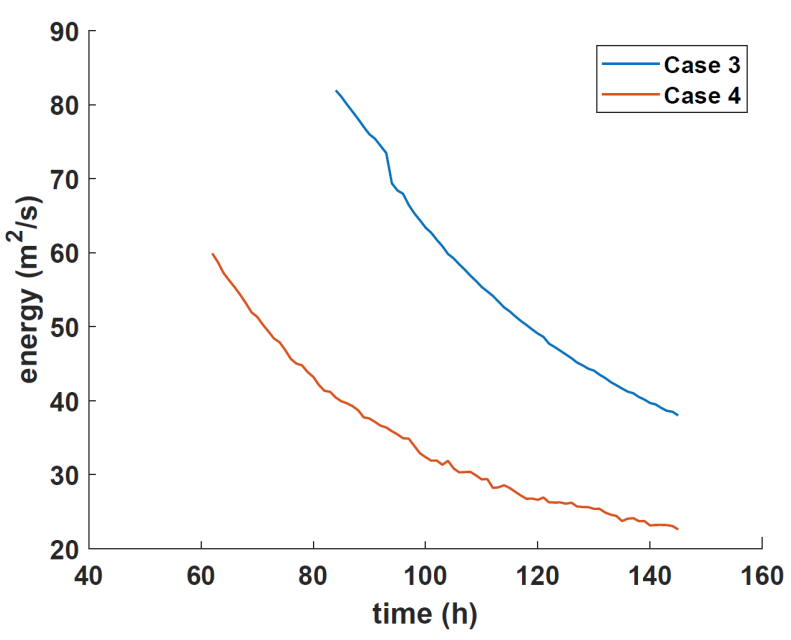

(b) Instance II.

Figure 18: Comparison of the time-energy Pareto-optimal curves for Case 3 and Case 4. 